\title{
A LUMINOUS, FAST RISING UV-TRANSIENT DISCOVERED BY ROTSE: A TIDAL DISRUPTION EVENT?
}

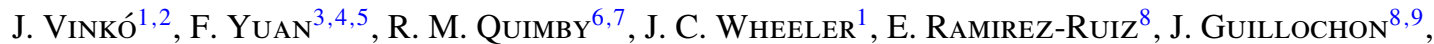 \\ E. Chatzopoulos ${ }^{1,10}$, G. H. Marion ${ }^{1}$, and C. AKerlof ${ }^{3}$ \\ ${ }^{1}$ Department of Astronomy, McDonald Observatory, University of Texas at Austin, Austin, TX 78712, USA; vinko@astro.as.utexas.edu \\ ${ }^{2}$ Department of Optics and Quantum Electronics, University of Szeged, Dóm tér 9, Szeged 6720, Hungary \\ ${ }^{3}$ Physics Department, University of Michigan, Ann Arbor, MI 48109, USA \\ ${ }^{4}$ Research School of Astronomy and Astrophysics, Australian National University, Weston Creek, ACT 2611, Australia \\ ${ }^{5}$ ARC Centre of Excellence for All-sky Astrophysics, Redfem, NSW 2016, Australia \\ ${ }^{6}$ Kavli IPMU, University of Tokyo, 5-1-5 Kahiwanoha, Kashiwa-shi, Chiba 277-8583, Japan \\ ${ }^{7}$ Department of Astronomy, San Diego State University, San Diego, CA 92182, USA \\ ${ }^{8}$ Department of Astronomy and Astrophysics, University of California, Santa Cruz, CA 95064, USA \\ ${ }^{9}$ Harvard-Smithsonian Center for Astrophysics, Cambridge, MA 02138, USA \\ ${ }^{10}$ FLASH Center for Computational Science, Department of Astronomy and Astrophysics, University of Chicago, Chicago, IL 60637, USA \\ Received 2014 June 15; accepted 2014 October 18; published 2014 December 12
}

\begin{abstract}
We present follow-up observations of an optical transient (OT) discovered by ROTSE on 2009 January 21. Photometric monitoring was carried out with ROTSE-IIIb in the optical and Swift in the UV up to +70 days after discovery. The light curve showed a fast rise time of $\sim 10$ days followed by a steep decline over the next 60 days, which was much faster than that implied by ${ }^{56} \mathrm{Ni}-{ }^{56} \mathrm{Co}$ radioactive decay. The Sloan Digital Sky Survey Data Release 10 database contains a faint, red object at the position of the OT, which appears slightly extended. This and other lines of evidence suggest that the OT is of extragalactic origin, and this faint object is likely the host galaxy. A sequence of optical spectra obtained with the $9.2 \mathrm{~m}$ Hobby-Eberly Telescope between +8 and +45 days after discovery revealed a hot, blue continuum with no visible spectral features. A few weak features that appeared after +30 days probably originated from the underlying host. Fitting synthetic templates to the observed spectrum of the host galaxy revealed a redshift of $z=0.19$. At this redshift, the peak magnitude of the OT is close to -22.5 , similar to the brightest super-luminous supernovae; however, the lack of identifiable spectral features makes the massive stellar death hypothesis less likely. A more plausible explanation appears to be the tidal disruption of a Sun-like star by the central supermassive black hole. We argue that this transient likely belongs to a class of super-Eddington tidal disruption events.
\end{abstract}

Key words: circumstellar matter - radiation mechanisms: non-thermal - stars: black holes - stars: magnetars supernovae: general

\section{INTRODUCTION}

In the past decade, untargeted ("blind") surveys revealed the existence of new types of transients. A good example is the case of superluminous supernovae (SLSNe): despite of being at least an order of magnitude brighter than "normal" supernovae (GalYam et al. 2009; Quimby et al. 2011; Gal-Yam 2012), SLSNe were not discovered before 2005, presumably because of the absence of their birthplaces (low-luminosity galaxies and/or galaxy cores) in the pre-selected target lists of earlier transient surveys (Quimby et al. 2011, 2013; Gal-Yam 2012).

The Texas Supernova Search (TSS; Quimby 2006) discovered the first two SLSNe, SN 2005ap (Quimby et al. 2007) and SN 2006gy (Smith et al. 2007), that became prototypes of two distinct subclasses within SLSNe (see Quimby et al. 2013 for details on the discoveries). Its successor, the ROTSE Supernova Verification Project (RSVP; Yuan 2010), continued to find SLSNe, e.g., SN 2008am (Chatzopoulos et al. 2011) and SN 2008es (Gezari et al. 2009). Both surveys extensively used the $0.45 \mathrm{~m}$ ROTSE-IIIb telescope at the McDonald Observatory, Texas. Although the target fields mostly covered rich galaxy clusters closer than $D \approx 200 \mathrm{Mpc}$, the majority of the transients discovered $(\approx 100$ to date) occurred in significantly more distant background galaxies. The details of the search and detection strategies are described in Quimby et al. (2012).

In this paper, we report the discovery of yet another unusual transient, detected with ROTSE-IIIb in the course of RSVP in 2009. The internal name of the transient was Dougie, but it has also been designated as ROTSE3J120847.9+430121. Although the early light curve (LC) and the first spectra taken with the 9.2 m Hobby-Eberly Telescope (HET) suggested a new SLSN, follow-up spectroscopic observations did not reveal any broad spectral features, which is unusual even among SLSNe that sometimes show peculiar spectral evolution. Instead, the spectra continued to show only a smooth, cooling continuum up to a month after discovery. At the last epochs when the transient was detected, narrow features due to the presumed host galaxy started to appear, and then the transient faded below the HET detection limit.

Subsequent spectroscopic observations with the Keck telescope confirmed the existence of the host galaxy at redshift of $z=0.19$. This redshift corresponds to a distance of $D=900 \mathrm{Mpc}$, which, when combined with photometric data, implies an observed absolute peak brightness of $M \approx-22.6 \mathrm{mag}$, similar to that of the brightest SLSNe (see Section 3.1).

Here we present a detailed account of the unique observational properties of Dougie as well as an in depth description of various model alternatives for its origin. This paper is organized as follows. In Section 2, the photometric and spectroscopic observations for both the transient and the host galaxy are presented. In Section 3, four alternatives for Dougie's origin are explored: a core-collapse supernova, an NS-NS merger, a gamma-ray burst (GRB) jet observed off-axis, and a tidal disruption of a low-mass stellar object by the central supermassive black hole, the latter of which is favored by the data. 


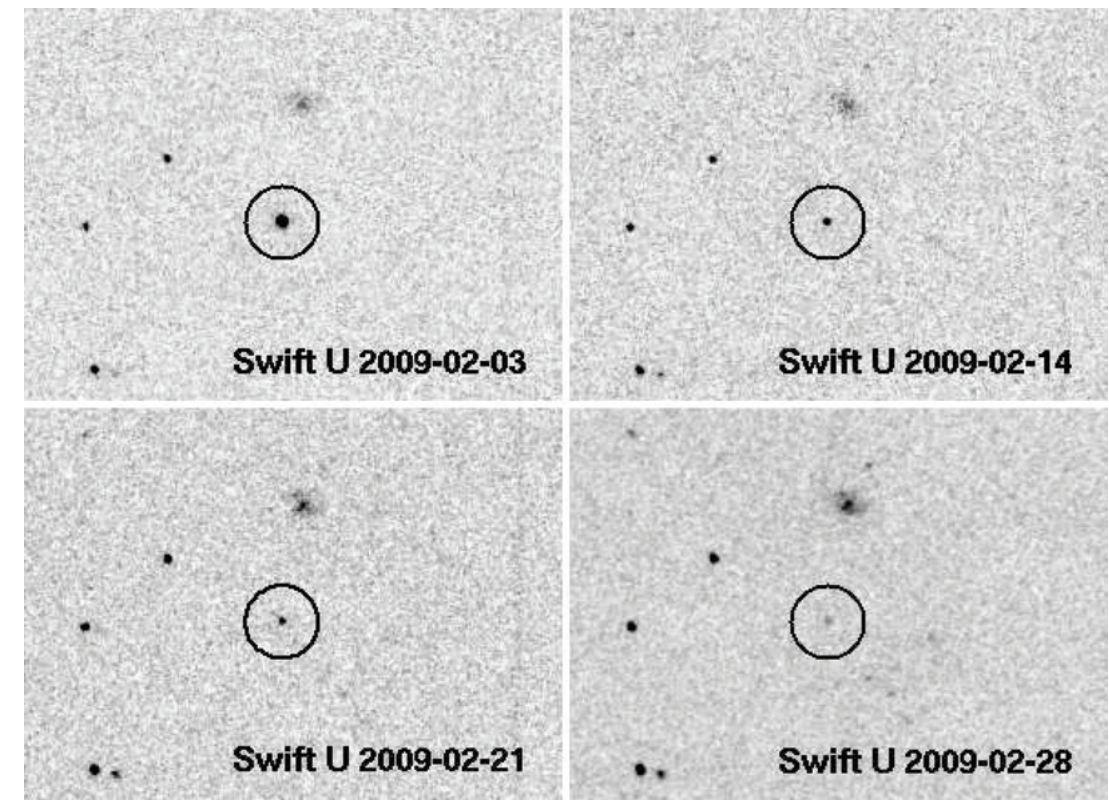

Figure 1. $5 \times 4 \operatorname{arcmin}^{2}$ fields of Swift/UVOT $u$-band frames around Dougie taken at different epochs.

Finally, in Section 4, we summarize our results and present our conclusions.

\section{OBSERVATIONS}

During its normal course of operation at the McDonald Observatory, Texas, ROTSE-IIIb detected a new object at R.A.= $12^{\mathrm{h}} 08^{\mathrm{m}} 47^{\mathrm{s}} .87\left( \pm 0.02^{s}\right)$, decl. $=+43^{\circ} 01^{\prime} 20^{\prime \prime} \cdot 1\left( \pm 0^{\prime} \cdot 09\right)$. The first detection occurred on MJD 54852.31 (2009 January 21 UT $07 \mathrm{~h}$ $26 \mathrm{~m} 24 \mathrm{~s}$; UT dates are used throughout this paper), supplemented by the next detection at UT $07 \mathrm{~h} 52 \mathrm{~m} 24 \mathrm{~s}$, confirming the presence of the new object. At the time of discovery, the apparent brightness of the transient was $\approx 17.3 \mathrm{mag}$ (all ROTSEIIIb unfiltered magnitudes have been converted to $R$-band magnitudes via USNO-B1.0 and Sloan Digital Sky Survey (SDSS) photometric calibrations; see Quimby et al. 2012). The ROTSE internal naming system identified the transient as Dougie. ${ }^{11}$

The position of the transient was checked in the SDSS Data Release 10 (DR10) catalog, and a very faint object, SDSS J120847.77+430120.1, was found at $\approx 1.4$ arcsec distance from Dougie. The object looks slightly more extended than nearby stars on the combined SDSS DR10 frame, thus, the SDSS pipeline classified this object as a galaxy and determined a photo- $z=0.207 \pm 0.017$ as the redshift estimate. Our subsequent spectroscopic observation (Section 2.5) confirmed the galaxy classification. We propose that this object is the host galaxy of the transient, and show below that our measurements support the likely extragalactic origin of Dougie.

\subsection{Photometry}

Tracing back in the ROTSE observational archive, the earliest detection of Dougie was found on the frames obtained at four days before discovery (MJD 54848.34, 2009 January 17) when the optical transient $(\mathrm{OT})$ was at $\approx 19.0 \mathrm{mag}$. The last pre-discovery non-detection (limiting magnitude $\approx 19.6 \mathrm{mag}$ ) occurred on 2009 January 15 (MJD 54846.3), six days before discovery. In the following, we assume that the outburst started

\footnotetext{
11 http://www.southparkstudios.com
}

during the two days between the last non-detection and the first successful detection, and set the "moment of first light" as $t_{0}=$ MJD $54847.3 \pm 1.0$ (hereafter we use the term "first light" to refer to the first observable appearance of the transient, thought to be the moment of shock breakout in $\mathrm{SNe}$, for example).

Photometric follow-up observations with ROTSE-IIIb began immediately after discovery, and continued up to 2009 February 15 when Dougie was at $\approx 18.5 \mathrm{mag}$.

Additional photometric data were collected by Swift/UVOT in three optical $(u, b, v)$ and three ultraviolet (UV) filters (uvw1, uvm2, uvw2) after triggering Swift in Target-of-Opportunity (ToO) observing mode. The UVOT observations started on 2009 January 28 and continued up to 2009 March 26, when the transient was below or close to the detection limit of UVOT in all filters. Figure 1 illustrates the temporal evolution of the OT on UVOT $u$-band frames.

Photometry of Dougie was computed applying aperture photometry on the Swift/UVOT Level-2 (sky) frames, using the calibration by Poole et al. (2008).

The photometry on the ROTSE-IIIb frames was performed by point-spread function (PSF) fitting on the template-subtracted frames. The results were converted to $R$-band magnitudes, as noted above. All photometric data are collected in Tables 1 and 2. The LCs are shown in Figure 2.

The redshift of Dougie's host galaxy was estimated spectroscopically (see Section 2.3) as $z=0.191$, which corresponds to a luminosity distance of $D_{L}=897 \mathrm{Mpc}$ assuming $\Lambda$-CDM cosmology with $H_{0}=73 \mathrm{~km} \mathrm{~s}^{-1} \mathrm{Mpc}^{-1}$. Using this distance, the observed peak $R$-band magnitude $(\approx 17.2 \mathrm{mag}$ ) translates to -22.6 mag absolute. As noted above, this peak brightness is comparable to that of the most luminous SLSNe.

\subsection{X-Ray Observations}

Dougie was monitored by Swift/XRT in X-rays between 0.2 and $10 \mathrm{keV}$, contemporaneously with the Swift/UVOT observations. A total of $21 \mathrm{ks}$ X-ray data were collected, extracted, and added up using the appropriate tools in HEAsoft.

Figure 3 shows a $5 \times 4$ arcmin field of the co-added XRT frame (after applying Gaussian smoothing) centered on the 


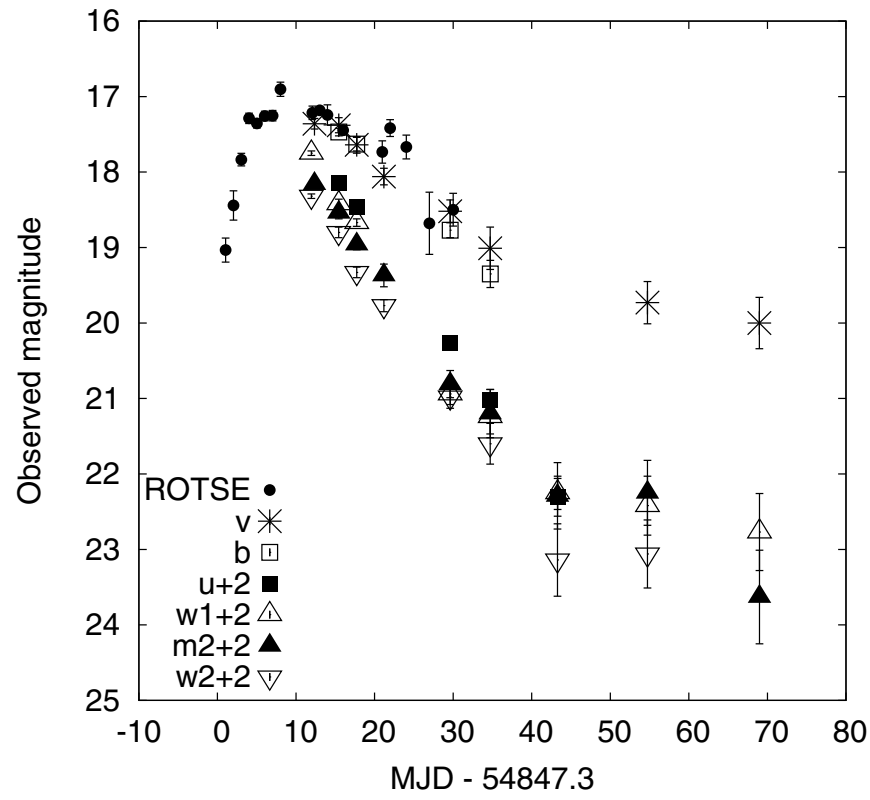

Figure 2. Light curves observed with ROTSE and Swift/UVOT. The UV data have been shifted down by 2 mag to enable comparison.

Table 1

ROTSE-IIIb Photometry of Dougie

\begin{tabular}{lccc}
\hline \hline MJD & $\begin{array}{c}R \\
(\mathrm{mag})\end{array}$ & $\begin{array}{c}\text { Error } \\
(\mathrm{mag})\end{array}$ & $\begin{array}{c}3 \sigma \text { limit } \\
(\mathrm{mag})\end{array}$ \\
\hline 54848.34 & 19.03 & 0.15 & 19.90 \\
54849.34 & 18.44 & 0.19 & 19.45 \\
54850.33 & 17.83 & 0.08 & 19.59 \\
54851.32 & 17.28 & 0.06 & 19.98 \\
54852.32 & 17.35 & 0.06 & 20.23 \\
54853.32 & 17.25 & 0.06 & 19.35 \\
54854.32 & 17.25 & 0.06 & 19.18 \\
54855.31 & 16.90 & 0.09 & 18.49 \\
54859.38 & 17.21 & 0.08 & 19.30 \\
54860.32 & 17.18 & 0.04 & 19.87 \\
54861.29 & 17.24 & 0.13 & 19.04 \\
54863.29 & 17.44 & 0.06 & 19.86 \\
54868.28 & 17.73 & 0.14 & 18.87 \\
54869.27 & 17.41 & 0.11 & 18.47 \\
54871.33 & 17.66 & 0.15 & 18.25 \\
54874.27 & 18.67 & 0.41 & 18.76 \\
54877.30 & 18.49 & 0.21 & 18.82 \\
\hline
\end{tabular}

Note. ${ }^{\text {a }}$ Without the $\approx 0.1 \mathrm{mag}$ zero-point uncertainty.

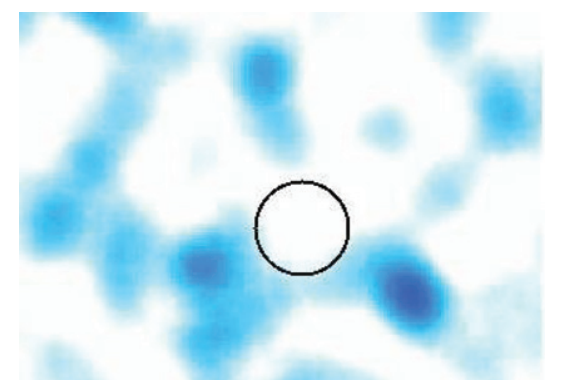

Swift XRT co-added 21 ks exposure

Figure 3. Swift/XRT co-added 21 ks exposure of the field around the expected position of Dougie. The field of view is the same as in Figure 1. No source is detected at the position of the transient.
Table 2

Swift/UVOT Photometry of Dougie

\begin{tabular}{|c|c|c|c|}
\hline MJD & Filter & $\begin{array}{c}m^{\mathrm{a}} \\
(\mathrm{mag})\end{array}$ & $\begin{array}{l}\text { error }^{\mathrm{b}} \\
\text { (mag) }\end{array}$ \\
\hline 54859.65 & $\mathrm{v}$ & 17.36 & 0.07 \\
\hline 54862.74 & $\mathrm{v}$ & 17.38 & 0.10 \\
\hline 54865.02 & $\mathrm{v}$ & 17.64 & 0.11 \\
\hline 54868.49 & $\mathrm{v}$ & 18.06 & 0.11 \\
\hline 54876.92 & $\mathrm{v}$ & 18.52 & 0.15 \\
\hline 54882.02 & $\mathrm{v}$ & 19.01 & 0.28 \\
\hline 54902.03 & $\mathrm{v}$ & 19.73 & 0.28 \\
\hline 54916.28 & $\mathrm{v}$ & 20.00 & 0.34 \\
\hline 54862.74 & $\mathrm{~b}$ & 17.47 & 0.05 \\
\hline 54865.03 & $\mathrm{~b}$ & 17.63 & 0.09 \\
\hline 54876.93 & $\mathrm{~b}$ & 18.77 & 0.10 \\
\hline 54882.03 & $\mathrm{~b}$ & 19.35 & 0.18 \\
\hline 54862.74 & $\mathrm{u}$ & 16.15 & 0.04 \\
\hline 54865.03 & $\mathrm{u}$ & 16.46 & 0.05 \\
\hline 54876.93 & $\mathrm{u}$ & 18.27 & 0.08 \\
\hline 54882.03 & $\mathrm{u}$ & 19.02 & 0.14 \\
\hline 54890.59 & $\mathrm{u}$ & 20.31 & 0.25 \\
\hline 54859.26 & uvw1 & 15.75 & 0.03 \\
\hline 54862.74 & uvw1 & 16.42 & 0.06 \\
\hline 54865.03 & uvw1 & 16.67 & 0.05 \\
\hline 54876.92 & uvw1 & 18.94 & 0.14 \\
\hline 54882.02 & uvw1 & 19.24 & 0.23 \\
\hline 54890.59 & uvw1 & 20.25 & 0.22 \\
\hline 54902.03 & uvw1 & 20.42 & 0.39 \\
\hline 54916.29 & uvw1 & 20.77 & 0.51 \\
\hline 54859.65 & uvm2 & 16.16 & 0.04 \\
\hline 54862.74 & uvm2 & 16.54 & 0.08 \\
\hline 54865.03 & uvm2 & 16.96 & 0.07 \\
\hline 54868.49 & uvm2 & 17.37 & 0.15 \\
\hline 54876.92 & uvm2 & 18.81 & 0.18 \\
\hline 54882.02 & uvm2 & 19.20 & 0.32 \\
\hline 54890.58 & uvm2 & 20.29 & 0.44 \\
\hline 54902.03 & uvm2 & 20.25 & 0.43 \\
\hline 54916.29 & uvm2 & 21.63 & 0.62 \\
\hline 54859.26 & uvw2 & 16.32 & 0.03 \\
\hline 54862.73 & uvw2 & 16.80 & 0.07 \\
\hline 54865.02 & uvw2 & 17.33 & 0.07 \\
\hline 54868.48 & uvw2 & 17.77 & 0.08 \\
\hline 54876.92 & uvw2 & 18.98 & 0.15 \\
\hline 54882.02 & uvw2 & 19.60 & 0.27 \\
\hline 54890.58 & uvw2 & 21.14 & 0.48 \\
\hline 54902.03 & uvw2 & 21.06 & 0.45 \\
\hline
\end{tabular}

Notes.

${ }^{\text {a }}$ Not corrected for host galaxy contamination.

${ }^{\mathrm{b}}$ Statistical uncertainty only.

position of Dougie. No source is detected at the position of the transient. Using WebPIMMS, ${ }^{12}$ the $3 \sigma$ detection limit, after correcting for the Galactic hydrogen column density of $N_{H}=1.28 \times 10^{20} \mathrm{~cm}^{-2}$ (Kalberla et al. 2005) and assuming $\gamma=1$ for the photon index, was found to be $f_{X}(3 \sigma)=6.88 \times$ $10^{-14} \mathrm{erg} \mathrm{s}^{-1} \mathrm{~cm}^{-2}$, corresponding to $L_{X}<6.6 \times 10^{42} \mathrm{erg} \mathrm{s}^{-1}$ for the upper limit of Dougie's X-ray luminosity.

\subsection{Spectroscopy}

Optical spectra were obtained with the Marcario LowResolution Spectrograph (LRS; Hill et al. 1998) mounted on

$\overline{12 \text { http://swift.gsfc.nasa.gov/Tools/w3pimms.html }}$ 
Table 3

Log of Spectroscopic Observations

\begin{tabular}{|c|c|c|c|c|c|c|c|c|}
\hline Date & MJD & $\begin{array}{l}\text { Phase }^{a} \\
\text { (days) }\end{array}$ & $\begin{array}{c}\text { Exposure } \\
\text { (s) }\end{array}$ & Airmass & $\begin{array}{c}\text { Range } \\
(\AA)\end{array}$ & $\begin{array}{c}\text { FWHM } \\
(\AA)\end{array}$ & $\mathrm{S} / \mathrm{N}^{\mathrm{b}}$ & Instr. \\
\hline 2009 Jan 24 & 54855.35 & +7 & 1800 & 1.16 & $4300-10,000$ & 19 & 42 & HET/LRS \\
\hline 2009 Jan 25 & 54856.32 & +8 & 1800 & 1.29 & $4300-10,000$ & 19 & 75 & HET/LRS \\
\hline 2009 Jan 29 & 54860.33 & +11 & 1800 & 1.20 & $4300-10,000$ & 19 & 75 & HET/LRS \\
\hline 2009 Jan 30 & 54861.50 & +12 & 600 & 1.04 & $3240-8950$ & 15 & 40 & P200/DBSP \\
\hline 2009 Feb 3 & 54865.30 & +15 & 1800 & 1.24 & $4300-10,000$ & 19 & 66 & HET/LRS \\
\hline 2009 Feb 7 & 54869.52 & +19 & 1800 & 1.19 & $4300-10,000$ & 19 & 44 & HET/LRS \\
\hline 2009 Feb 15 & 54877.26 & +25 & 1800 & 1.27 & 4300-9000 & 19 & 32 & HET/LRS \\
\hline 2009 Feb 20 & 54882.50 & +30 & 1800 & 1.24 & $4300-10,000$ & 19 & 39 & HET/LRS \\
\hline 2009 Feb 26 & 54888.46 & +35 & 1800 & 1.16 & $4250-10,000$ & 19 & 15 & HET/LRS \\
\hline 2009 Mar 1 & 54891.23 & +37 & 5100 & 1.15 & $4300-10,000$ & 19 & 43 & HET/LRS \\
\hline 2009 Mar 2 & 54892.22 & +38 & 3600 & 1.31 & 4200-9100 & 19 & 14 & HET/LRS \\
\hline 2009 Nov 11 & 55146.60 & +251 & $900 / 765$ & 1.63 & $3300-10,000$ & 6 & 11 & Keck-I/LRIS \\
\hline
\end{tabular}

Notes.

${ }^{a}$ Rest-frame days since outburst assuming $T_{0}=54847.3 \mathrm{MJD}$ and $z=0.191$.

b Signal-to-noise measured at $6000 \AA$.

the $9.2 \mathrm{~m}$ HET (Ramsey et al. 1998) at the McDonald Observatory, Texas. Ten spectra were collected between 2009 January 24 and March 2, starting around maximum light and extending up to about one month thereafter. In addition, a spectrum was taken with the Double Spectrograph (DBSP; Oke \& Gunn 1982), operating on the Palomar 200 inch telescope, on 2009 January 30. The log of the spectral observations is presented in Table 3.

All spectra were reduced in the standard way using IRAF. ${ }^{13}$ Wavelength calibration was done based on combined exposures of $\mathrm{Cd}$ and $\mathrm{Ne}$ spectral lamps. Flux calibration was computed using spectra of spectro-photometric, flux standard stars taken on the same nights when the transient was observed, which provided reliable relative fluxes for the object's spectra. Absolute flux levels were determined by matching the HET spectra with the flux densities from contemporaneous Swift/UVOT $b$ and $v$ observations.

The observed spectral sequence is plotted in Figure 4, where the individual spectra have been shifted vertically for clarity and also Doppler-corrected back to the host galaxy's rest frame assuming $z=0.191$ (see below).

The spectra are dominated by a smooth, hot continuum without any obviously noticeable spectral feature. At later phases (after February 20), weak narrow features appeared between 4000-6000 Å rest-frame wavelengths, which are probably due to contamination from the host galaxy (see Section 2.3).

The combined optical + UV spectral energy distribution (SED) of Dougie was constructed by combining the HET spectra with the Swift/UVOT photometric flux densities taken close to the spectroscopic observations. These SEDs were then corrected for Milky Way extinction using $E(B-V)_{\text {gal }}=0.0136$ (Schlegel et al. 1998). Reddening within the host galaxy was ignored because of the lack of information on this parameter, but the very blue observed color of the transient during the early phases argues against significant in-host extinction. Finally, the flux contribution from the host galaxy was also subtracted from the combined UV-optical SEDs. This correction was negligible during the early phases, but increased considerably when the transient evolved after maximum.

13 IRAF is distributed by the National Optical Astronomy Observatories, which are operated by the Association of Universities for Research in Astronomy, Inc., under cooperative agreement with the National Science Foundation.

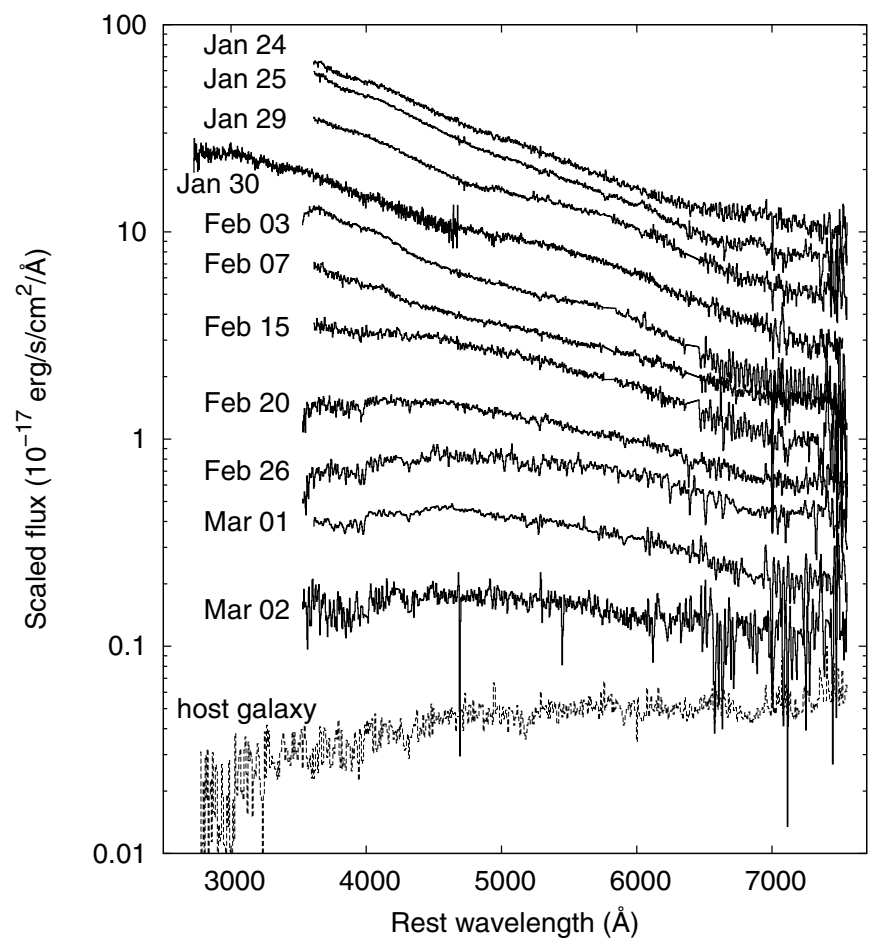

Figure 4. Sequence of observed optical spectra, corrected for redshift $(z=$ 0.191 ; see the text), and shifted vertically for better visibility. Rest-frame phases since the estimated moment of outburst (see Table 3 ) are indicated by the labels next to each spectrum. The last spectrum is that of the host galaxy (Section 2.5). All spectra are dominated by a cooling continuum, and do not show any obvious spectral feature.

Figure 5 plots the temporal evolution of the SED in rest-frame days. The SED peak is observed to gradually shift from $2200 \AA$ at $+10 \mathrm{~d}$ to $4600 \AA$ at $+36 \mathrm{~d}$, corresponding to $\approx 13,000 \mathrm{~K}$ and $\approx 6300 \mathrm{~K}$ Wien-temperatures, respectively. The flux depression between $2500-3000 \AA$ appearing after $+25 \mathrm{~d}$ might be due to the broad UV-features observed in the spectra of the SLSN PS110bzj (Lunnan et al. 2013) and the TDE candidate PS1-11af (Chornock et al. 2014); however, the resolution provided by the broadband Swift UV filters are not adequate to unambiguously identify these features. Alternatively, the "UV-bump" appearing on $+36 \mathrm{~d}$ might be caused by the red leak of the Swift UV-filters. 


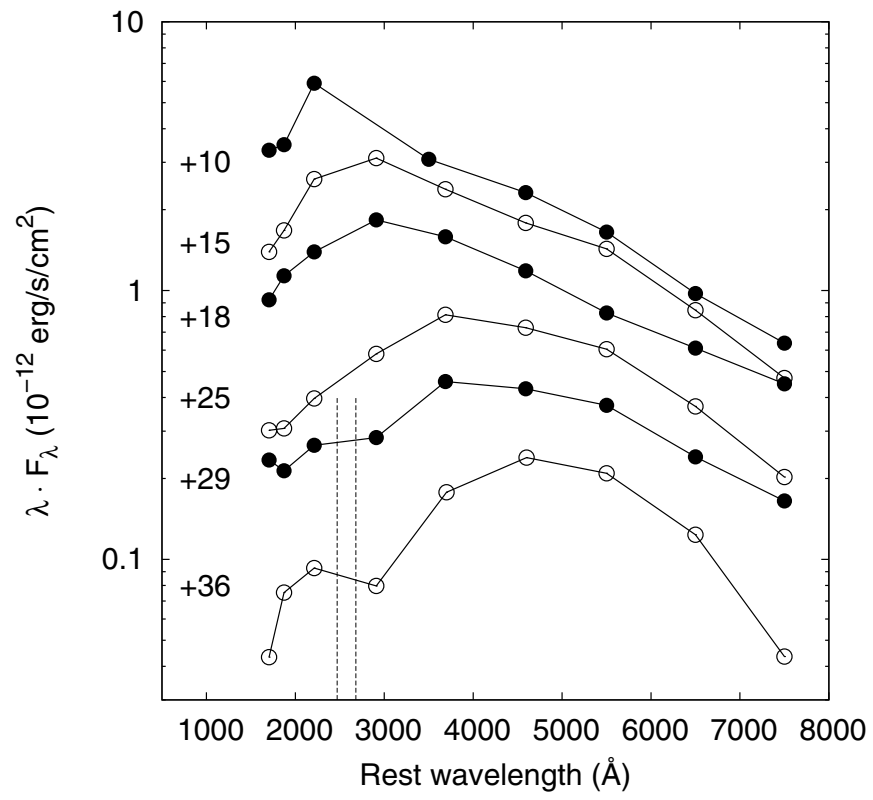

Figure 5. Evolution of the optical + UV SED after correcting for host galaxy contamination. The phase of the transient (the elapsed time since outburst in rest-frame days) is indicated at the left-hand side of each SED. No vertical shifts have been applied between the curves. The dotted vertical lines mark the position of the $2470 \AA$ and the $2680 \AA$ features observed in PS1-10bzj (Lunnan et al. 2013) and PS1-11af (Chornock et al. 2014).

The SEDs in Figure 5 cannot be described by a series of single-temperature blackbodies: the optical continuum may suggest a higher temperature, but the increasing flux decline in the UV is inconsistent with the hot blackbody assumption. It is possible that the UV is affected by strong blending due to ionized metal lines, as usual in $\mathrm{SNe}$; however, without having a well-resolved UV spectrum, such a conclusion cannot be proven unambiguously.

\subsection{Comparison with Spectra of SLSNe}

The earliest spectra of Dougie appeared similar to those of some SLSNe observed with HET, showing mostly a hot, featureless continuum. This is illustrated in the left panel of Figure 6, where the January 25 spectrum (+8 day phase after first light in rest-frame) is plotted together with the early-phase HET spectra of two H-rich SLSNe: SN 2008am (Chatzopoulos et al. 2011) and SN 2008es (Gezari et al. 2009). It is clearly observed that unlike SN 2008am, Dougie did not show either hydrogen or any other spectral features. SN 2008es was similarly absent of features in the early spectra, but its late-time spectra (not shown here) contained strong, unambiguous SN features including Balmer lines (Gezari et al. 2009; Miller et al. 2009). On the contrary, as seen in Figure 4, none of Dougie's observed spectra show SN-like features.

Figure 6 also illustrates that the continuum slope of Dougie's early-phase optical spectra is relatively well described by a power law with $F_{\lambda} \propto \lambda^{-3}$. Pure, hotter blackbody spectra are incompatible with the UV SED. The observed spectra of Dougie cannot be accurately modeled by either an evolving single-temperature blackbody or by a power-law spectrum with a fixed slope.

The right panel of Figure 6 shows a similar comparison between the last observed spectrum of Dougie ( +37 days after first light or +30 days after the peak of the LC, both measured in rest-frame) and spectra of SN 2010kd (J. Vinko et al., in preparation), and PTF12dam (Nicholl et al. 2013; Chen et al. 2014). Contrary to the early-phase spectrum in the left panel, this late-phase spectrum of Dougie is redder than the other SLSNe at similar post-peak phases. This and the lack of the spectral features make the spectral evolution of Dougie being quite different from both H-rich and H-free SLSNe.

We cannot rule out that the lack of broad SN features in Dougie's spectra might be simply due to an observational effect. The broad SN features might have appeared only at later times when the transient faded below the HET detection limit. Although this scenario cannot be excluded, this seems improbable if Dougie is thought to be similar to other fastevolving SLSNe, like SN 2008es, which occurred at a similar distance. In SN 2008es the broad SN features started to appear after +20 rest-frame days (Miller et al. 2009), while in Dougie they failed to appear for at least +38 rest-frame days.

Similar hot, featureless spectra have also been observed in more recent SLSNe such as PS1-10bzj (Lunnan et al. 2013) and CSS121015 (Benetti et al. 2014); but, again, there are important differences in the observed spectral evolution between these SLSNe and Dougie. CSS121015 was a slowly evolving H-rich SLSN (its LC peaking at $+40 \mathrm{~d}$ rest-frame), which developed broad H, Ca II, Mg II, and Fe II features after $+100 \mathrm{~d}$ (Benetti et al. 2014). By contrast, PS1-10bzj was an H-poor SLSN showing rapid evolution, and by $+16 \mathrm{~d}$ rest frame it had also developed the usual broad features common to all H-poor SLSNe (Lunnan et al. 2013). Although SLSNe show some degree of diversity in their observed properties, the lack of any broad spectral feature in Dougie's spectra during the entire observable window is unprecedented to date.

\subsection{The Host Galaxy}

The candidate host for Dougie is the galaxy SDSS J120847.77+430120.1. The SDSS ugriz PSF AB-magnitudes for this object are $u^{\prime}=23.096( \pm 0.428), g^{\prime}=21.486(0.052)$, $r^{\prime}=20.299(0.025), i^{\prime}=19.882(0.036)$, and $z^{\prime}=19.510$ (0.059), while its photo- $z$ is $0.207 \pm 0.017$ according to the SDSS DR10 database.

There is no detected object in the Galaxy Evolution Explorer $(G A L E X)$ database $^{14}$ closer than 10 arcsec to this position. Adopting $m_{\mathrm{AB}}=20.5 \mathrm{mag}$ as the limiting magnitude for the GALEX all-sky survey, the background-corrected flux upper limit for the host is $\approx 1.3 \times 10^{-16} \mathrm{erg} \mathrm{s}^{-1} \mathrm{~cm}^{-2} \AA^{-1}$ in both the $\operatorname{NUV}(\lambda 2271 \AA)$ and the FUV ( $\lambda 1528 \AA)$ bands. The lack of UV detection is consistent with the photometric and spectroscopic optical observations (see below). Also, there is no known $\mathrm{X}$-ray or radio source in the vicinity of Dougie's position. According to the SIMBAD ${ }^{15}$ database, the closest radio source (WN J1208+4301) is $\sim 1$ arcmin away and is not related to the host.

We have observed the candidate host galaxy with the doublechannel Low Resolution Imaging Spectrometer (LRIS) (Oke et al. 1995) mounted on the Keck I telescope on 2009 Nov 11.6 UT (MJD 55146.60). The spectrum is plotted together with the broadband SDSS fluxes in Figure 7. A Sb-type galaxy template taken from Kinney et al. (1996) is also shown for comparison.

Cross-correlation between the observed and the template galaxy spectra revealed $z=0.191 \pm 0.022$ as the optimum estimate for the redshift of the host, which is adopted for this

\footnotetext{
14 http://galex.stsci.edu/GR6/

15 http://simbad.u-strasbg.fr
} 

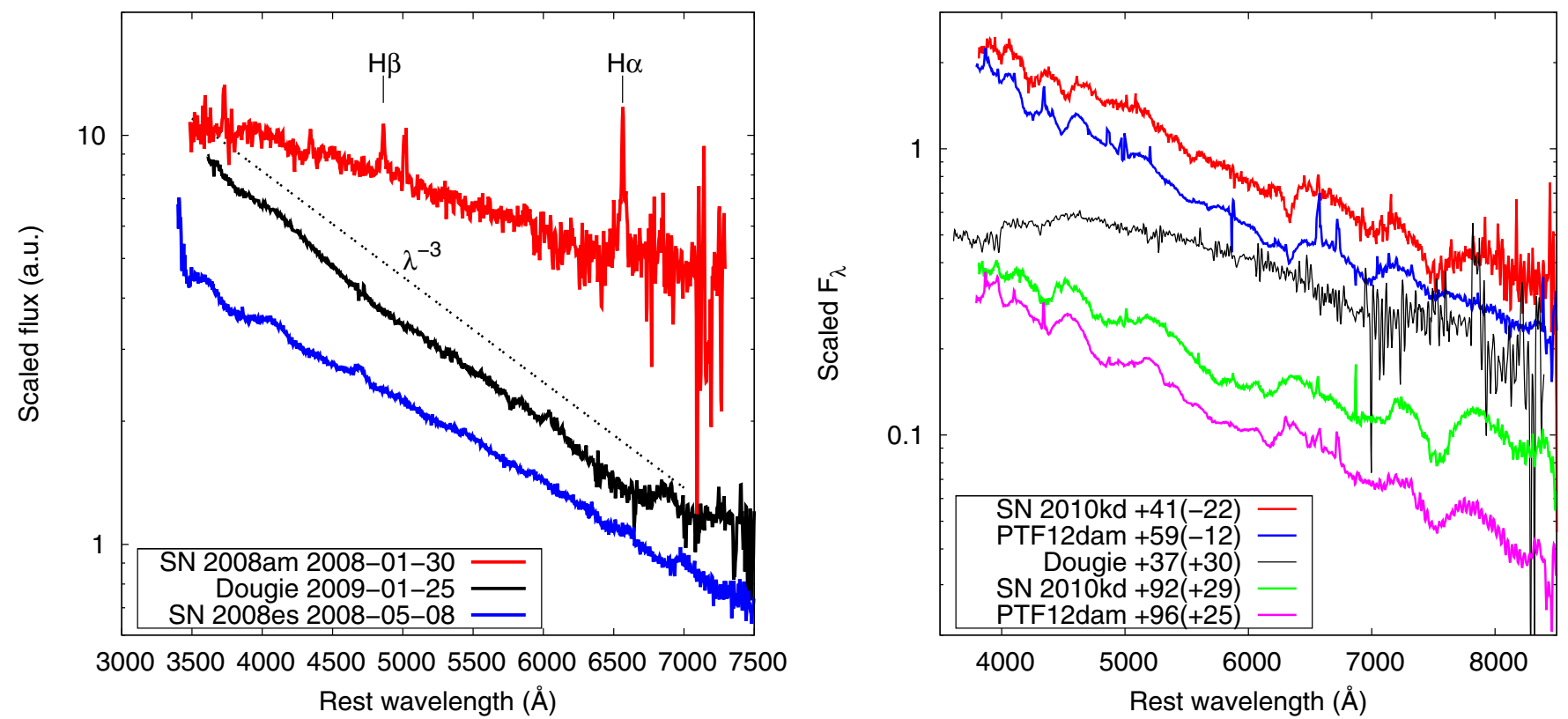

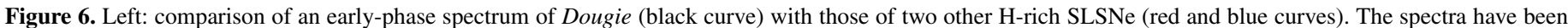

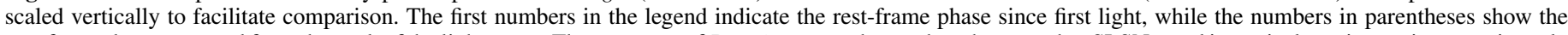

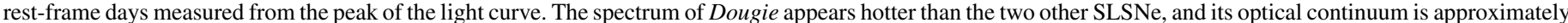

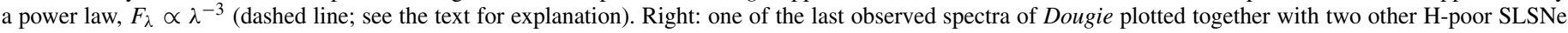
observed with HET at similar phases.

paper. This spectroscopic redshift is consistent with the photo- $z$ estimate derived from the SDSS photometry.

Note that there is no indication for either the presence of an active galactic nucleus (AGN) or any ongoing star formation in the host galaxy spectrum. These would produce strong, unambiguous, narrow emission lines that are not observed in the galaxy spectrum.

\subsection{Position within the Host Galaxy}

The bright, fast-rising transient appeared slightly offset from the centroid of the host galaxy as reported in the SDSS DR10 catalog. Since the position of Dougie within the host galaxy can be key for interpreting its physical origin, here we investigate this issue in more detail.

In order to estimate the uncertainties associated with Dougie's position, we first co-added Swift/UVOT frames obtained on January 31 and February 3 (+10 and +15 rest-frame days, respectively), when the OT was observed with the highest signal to noise. We then register the Swift frames to the SDSS $r$-band frame of the same area by matching the positions of common point sources on both frames. Next, we determine the coordinates of Dougie on the registered Swift frames and compared them to those of the photometric centroid of the host galaxy as measured on the SDSS $r$-band frame. The results are shown in Figure 8, where the Swift minus SDSS coordinate differences (in arcseconds) are plotted as $\Delta$ R.A. and $\Delta$ decl. Open symbols represent the coordinate differences for the reference objects, while the filled symbols denote the position of Dougie with respect to the host photo-center.

Figure 8 reveals that that the reference objects scatter around the zero point more or less isotropically, and there is no obvious systematic shift between the data obtained on the epochs of the two Swift observations. On the other hand, Dougie appears to be systematically offset, about $11^{\prime \prime} 2$ east and 0 .'4 south from the

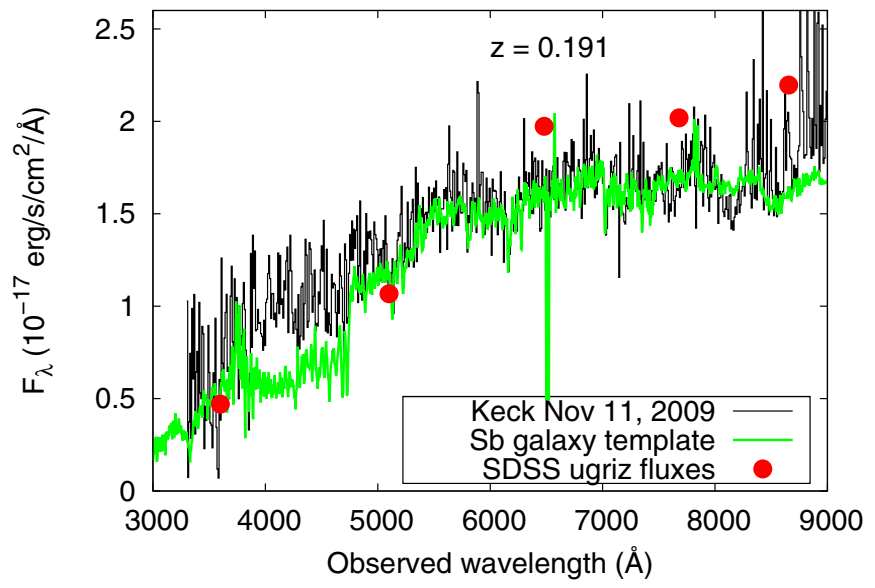

Figure 7. Observed Keck spectrum of the host (black curve) compared with an Sb-type galaxy template (green curve) redshifted to $z=0.191$. The ugriz fluxes (red filled circles) from SDSS DR10 are also overplotted. The host spectrum appears to be dominated by older stellar population and does not show any sign of either enhanced star formation or AGN activity.

center of the host, in both observed epochs. Adopting the WCS of the SDSS frame, we measure the final position of Dougie as R.A. $=12^{\mathrm{h}} 08^{\mathrm{m}} 47^{\mathrm{s}} .86( \pm 0.02)$, decl. $=+43^{\circ} 01^{\prime} 19^{\prime \prime} .9\left( \pm 0^{\prime \prime} .6\right)$. We note that the uncertainties for Dougie plotted in Figure 8 are conservative \pm 1 pixel errors of the Swift frames that may overestimate the true errors of the photo-centers. The coordinate uncertainties given above are in between the \pm 1 pixel errors and the measured frame-to-frame shift of the photometric center of the OT ( $\sim 0.1$ pixel) on the Swift frames.

Based on our Swift observations, we infer that Dougie appears to be slightly off-center from its host galaxy. The total angular distance from the photo-center of the host is $\approx 1.3$ arcsec, 


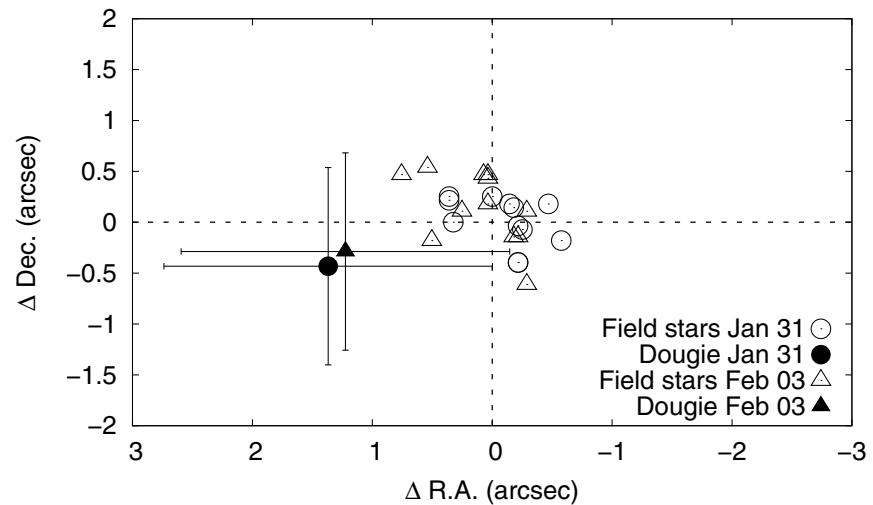

Figure 8. Position of the transient (filled symbols) as measured on the January 31 (circle) and February 3 (square) Swift $U$-band frames relative to the photometric centroid of the host galaxy on the $r$-band SDSS frame. The error bars represent 1 pixel uncertainty of the centroid on the Swift frames (see the text). Open circles and triangles denote the position differences for the reference objects on the same Swift and SDSS frames. The scattering of the open symbols around the center illustrate the registration uncertainty between the Swift and SDSS World Coordinate Systems. The transient appears $1.3 \operatorname{arcsec}(3.9 \mathrm{kpc})$ off the photo-center of the host.

corresponding to $\approx 3.9 \mathrm{kpc}$ linear distance for the adopted redshift $(z=0.191$, Section 2.4).

\subsection{The Quasi-bolometric Light Curve}

The bolometric LC of the transient was assembled by integrating the host-corrected UV+optical SEDs (Figure 5) over wavelength. The fluxes in the far-UV were estimated by linearly extrapolating the spectrum until $1000 \AA$. In the IR (longward of $8000 \AA$ ), a Rayleigh-Jeans tail starting from the reddest observed flux was assumed to calculate the integrated IR contribution to the total quasi-bolometric flux.

The temporal coverage of the LC was refined by adding more data points from the ROTSE $R$-band and Swift $v$-band LCs (Figure 2). This was justified by the close similarity between the shape of the LCs at optical wavelengths. To do this, the observed ROTSE and Swift magnitudes were converted to absolute magnitudes using a $D_{L}=897 \mathrm{Mpc}$ luminosity distance, then the luminosity curve was calculated from these absolute magnitudes as if they were bolometric magnitudes, which is equivalent to assuming $\mathrm{BC}=0 \mathrm{mag}$ bolometric correction. Although this seems like a rather crude approximation, the resulting LC (plotted in Figure 9) appears to be consistent with those obtained from SED integration. The peak bolometric luminosity of Dougie is thus estimated to be $L_{\text {peak }} \approx 5( \pm 1) \times 10^{44} \mathrm{erg} \mathrm{s}^{-1}$, while the integrated radiated energy is calculated to be $E_{\text {rad }} \approx 6.2( \pm 0.2) \times 10^{50} \mathrm{erg}$.

\section{ON THE ORIGIN OF DOUGIE}

In this section, we consider four models for the origin of Dougie: core collapse supernova, merging neutron stars, off-axis GRB models, and the tidal disruption of a star by the central supermassive black hole. These are presented in detail in the following subsections.

\subsection{Core Collapse Models}

Although the lack of SN features in the optical spectra does not support the core collapse hypothesis, the LC appears rather similar to those of SLSNe. For this reason, in the absence of spectral information, one could have naturally deduced that Dougie might have resulted from the death of a massive star that was recently formed in the host galaxy.

In the left panel of Figure 9, we compare the bolometric LC of Dougie with those of several SLSNe. The data of the latter objects were analyzed by Chatzopoulos et al. (2011), where the reader may find the references to the data. It is seen that Dougie showed faster LC evolution than most of the well-observed SLSNe. The rise-time to peak, $t_{\text {rise }} \sim 10$ days, was similar to that of PTF09uj, a luminous Type IIn SN (Ofek et al. 2010). However, as Figure 9 shows, the peak luminosity of Dougie clearly puts it into the SLSN regime.

In this subsection, we make an effort to describe the LC using simple $\mathrm{SN}$ radiative diffusion models to ascertain whether or not they can provide a reasonable description of the bolometric LC. Following the formalism developed by Chatzopoulos et al. (2011), we test three different core collapse scenarios: a Ni-Co radioactive decay model, a magnetar model (Kasen \& Bildsten 2010; Woosley 2010), and a shocked, circumstellar medium (CSM) model. The best-fit representations of the LC are plotted
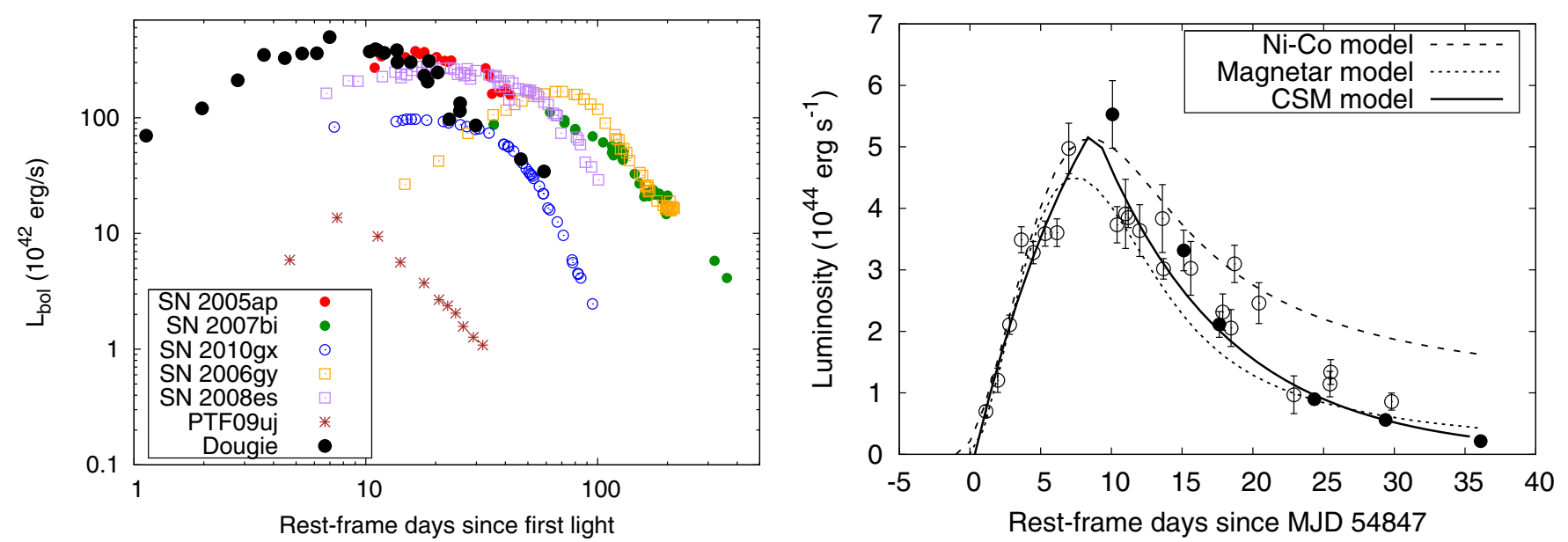

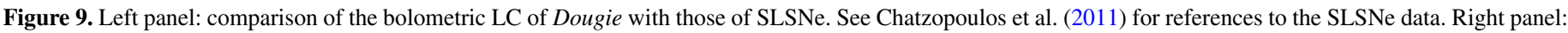

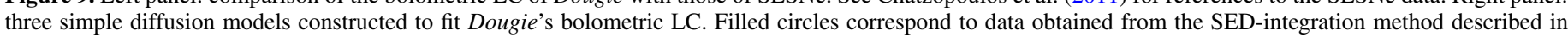

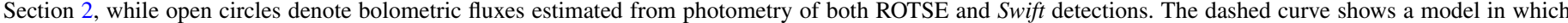

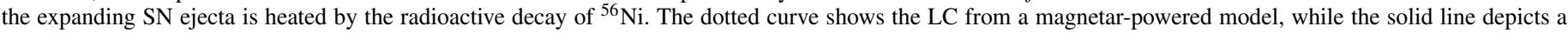
shocked, CSM model. The reader is referred to Section 3.1 for a brief discussion of the model parameters. 
in Figure 9. All three models assume that the energy is deposited at the center of an optically thick sphere, promptly thermalized, and then slowly transported out by photon diffusion. In the radioactive decay and magnetar modes, we assume homologous expansion of the SN ejecta (which is taken into account when solving the diffusion equation), while in the CSM model we assume a fixed, opaque CSM cloud whose interior is thermalized at a designated time.

The fast rise and decay of the observed LC can be fit only using a relatively short effective diffusion timescale, which corresponds to a low ejecta mass in all three scenarios. The high peak luminosity, on the other hand, requires very large internal energy to be readily available. In the radioactive decay model, in particular, the derived diffusion timescale, $t_{\mathrm{d}} \approx 7-8$ days, implies $M_{\mathrm{ej}} \approx 1 M_{\odot}$ for $\kappa=0.1 \mathrm{~cm}^{2} \mathrm{~g}^{-1}$ and $v_{\mathrm{sn}}=3 \times$ $10^{4} \mathrm{~km} \mathrm{~s}^{-1}$, while the large peak luminosity demands a Nimass $\approx 15 M_{\odot}$. In the magnetar model, the early peak requires a short ( $\sim 5$ days) spin-down timescale, which in turn requires a relatively large magnetic field strength of about $4 \times 10^{14} \mathrm{G}$ for an initial $10 \mathrm{~ms}$ spin period. Moreover, the magnetar model also needs a relatively low-mass ejecta $\left(M_{\mathrm{ej}} \sim 1 M_{\odot}\right)$, but a very extended initial radius of $R_{0} \sim 10^{14} \mathrm{~cm}$. In addition, this model requires the internal energy to be injected near the edge of the very tenuous SN ejecta to avoid significant adiabatic degradation.

The CSM model gives a more physically consistent picture with a required total shocked energy of about $8 \times 10^{50} \mathrm{erg}$ (compatible with the explosion energy of a typical SN) deposited within a CSM cloud of about $\approx 2.6 M_{\odot}$. In this scenario, Dougie resembles to PTF09uj (Ofek et al. 2010; Chatzopoulos et al. 2011), i.e., the luminosity is due to the conversion of shock kinetic energy into thermal energy within the opaque, dense CSM. The higher luminosity of Dougie might be explained by the larger kinetic energy and denser, more massive CSM than in the case of PTF09uj. However, the weakness of this hypothesis is that, unlike in PTF09uj, there is no indication for any shock-generated emission lines in the spectra of Dougie, which are ubiquitously observed in interacting Type IIn SNe. In principle, the lack of hydrogen and/or helium emission lines might be consistent with the presence of a $\mathrm{H} / \mathrm{He}$-free, O-rich CSM around the transient, but the lack of any kind of spectral feature during the whole observed period makes this hypothesis less feasible.

The other important observational constraint that argues against the core-collapse SLSN scenario is the nature of the host galaxy (Section 2.5). There is growing evidence that $\mathrm{H}$-free SLSNe tend to appear in metal-poor dwarf galaxies that show intense star formation rates and extremely strong emission lines (Neill et al. 2011; Lunnan et al. 2014; Leloudas et al. 2014). Since the host of Dougie appears to be dominated by older populations of stars without any sign of enhanced star formation, these observed properties of the host galaxy strongly argue against the SLSN nature of Dougie.

\subsection{Neutron Star Merger Model}

Merging neutron stars (NS-NS mergers, or "merger-novae") are thought to be promising candidates for producing fast, luminous transients (Lee \& Ramirez-Ruiz 2007; Metzger et al. 2010; Roberts et al. 2011; Barnes \& Kasen 2013; Yu et al. 2013; Metzger \& Piro 2014). Recently, the rapid OT PTF11agg (Cenko et al. 2013) was proposed to be due to such a phenomenon ( $\mathrm{Wu}$ et al. 2014). In this model, the merging of two neutron stars due to gravitational wave radiation is speculated to produce, in some cases, a rapidly spinning, hypermassive, magnetized neutron star (Rosswog et al. 2003) surrounded by a more or less spherical, fast-expanding $(v \sim 0.1 c)$ envelope, the mass of which is $M_{\mathrm{ej}} \lesssim 0.1 M_{\odot}$. The magnetar wind is assumed to efficiently dissipate Poynting flux within the expanding envelope, heating it and accelerating it to relativistic speed $(v \sim c)$. The dilution of the envelope due to expansion causes the thermalized photons to escape on timescales anywhere between hours and days depending primarily on the mass of the surrounding envelope and its expansion velocity. Thus, the basic physical configuration, to some extent, is similar to the magnetar model considered in Section 3.1. The main difference being the mass of the ejected envelope, which in the SN model is at least an order of magnitude larger.

While the stable, hypermassive neutron stars model could produce LCs with shapes that are qualitatively similar to that of Dougie, i.e., rapid rise followed by slower decline, the calculations by, e.g., Yu et al. (2013) and Metzger \& Piro (2014) show rise times that are significantly shorter (typically between $10 \mathrm{hr}-1$ day) than the $\sim 10$ days observed rise time of Dougie. This is essentially due to the smaller ejected mass in the NS-NS merger systems and its corresponding higher expansion velocity. As it was shown in Section 3.1, the observed LC of Dougie needs $t_{\mathrm{d}} \sim 8$ days, which is almost a factor of 10 longer than the typical diffusion timescales expected in NS-NS merger systems. Therefore, we conclude that the predictions of the merger model are not compatible with the observed LC of Dougie.

\subsection{Orphan Afterglow Model}

Given that most GRBs are collimated into narrow jets, their observed properties will unavoidably vary depending on the angle $\theta_{\text {obs }}$ from their symmetry axis at which they are observed. If Dougie were a GRB, then at least its gammaray emission directed at us was certainly extremely weak. A plausible interpretation might be that the Dougie was a typical GRB seen at an angle, $\theta_{\text {obs }}$, larger than the opening angle of the central jet, $\theta_{\mathrm{j}}$.

If we assume a homogeneous sharp-edged jet, the burst seen by all observers located within the initial jet aperture, $\theta_{\mathrm{obs}}<\theta_{\mathrm{j}}$, is practically the same, but beyond the edges of the jet the emission declines precipitously (Woods \& Loeb 1999; Granot et al. 2002; Ramirez-Ruiz et al. 2005). When $\theta_{\text {obs }} \gg \theta_{\mathrm{j}}$, there is no detectable prompt emission and the accompanying early afterglow is weak, owing to relativistic beaming of photons away from the line of sight. As the Lorentz factor decreases with time, an off-axis observer will see a rising afterglow LC at early times peaking when the jet Lorentz factor reaches $\approx 1 /\left(\theta_{\text {obs }}-\theta_{\mathrm{j}}\right)$ and approaching that seen by an on-axis observer at later times. This is because an observer will receive most emission from those portions of a GRB blast wave that are within an angle $1 / \Gamma$ to the direction to the line of sight such that the emission for an offaxis observer will remain at a very low level until the Doppler cone of the beam intersects the observer's line of sight. This can be seen by comparing the $\theta_{\mathrm{obs}}<\theta_{\mathrm{j}}$ and $\theta_{\mathrm{obs}}=1.6 \theta_{\mathrm{j}} \mathrm{LCs}$ in Figure 10.

The off-axis GRB interpretation of Dougie requires the viewing angle to have been $\theta_{\mathrm{obs}} \approx 1.6 \theta_{\mathrm{j}}$ (Figure 10), similar to the parameters determined by Cenko et al. (2013) for PTF11agg. The afterglow LCs at $v \approx 4.5 \times 10^{14} \mathrm{~Hz}$ presented here are calculated using the afterglow models of van Eerten et al. (2012) 


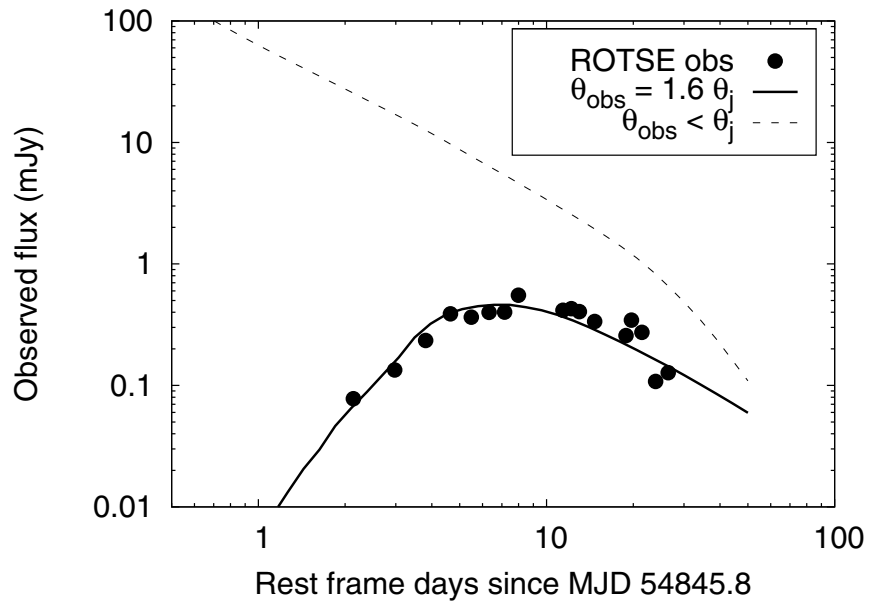

Figure 10. Afterglow emission from an off-axis GRB jet. Light curves calculated for two viewing angles $\theta_{\mathrm{obs}} \leqslant \theta_{\mathrm{j}}$ (dashed curve) and $\theta_{\mathrm{obs}}=1.6 \theta_{\mathrm{j}}$ (solid curve), and for a GRB with $\theta_{\mathrm{j}}=0.3, E_{\Omega}=10^{54} \mathrm{erg}, p=2.5, n_{0}=1.0 \mathrm{~cm}^{-1}$, acceptable match to the ROTSE unfiltered magnitudes (filled symbols), which correspond to $v \approx 4.5 \times 10^{14} \mathrm{~Hz}$.

by applying the BOXFIT ${ }^{16}$ code. In these models, the expansion of the GRB jet in a uniform medium with density $n_{0}$ is calculated using relativistic hydrodynamical simulations (e.g., Zhang \& MacFadyen 2009; De Colle et al. 2012a; van Eerten et al. 2010; De Colle et al. 2012c). The local emissivity is computed using the conventional assumptions of synchrotron emission from relativistic electrons that are accelerated behind the shock into a power-law distribution $\left(\propto \gamma^{-p}\right)$, where the electrons and the magnetic field hold fractions $\epsilon_{e}$ and $\epsilon_{B}$, respectively, of the internal energy of the shocked fluid (Sari et al. 1998).

One question that naturally arises is whether the observed multi-wavelength evolution can be explained within the framework of this model. Even though the model fits the optical LCs moderately well, it is inconsistent with the observed SED and, in addition, predicts significantly higher X-ray fluxes when compared with the Swift/XRT upper limits. This is illustrated in Figure 11, where the predicted synchrotron spectra (solid lines) are compared with the observed SED. This argues against an off-axis GRB origin for Dougie.

\subsection{TDE Models}

The tidal disruption of an approaching star by a supermassive black hole (SMBH) has become a popular mechanism for explaining the bright, slowly evolving X-ray/UV/optical "flares" from luminous transients (see, e.g., Chornock et al. 2014; Arcavi et al. 2014; Holoien et al. 2014, and references therein). TDEs are characterized by the following basic quantities: SMBH mass $M_{\mathrm{h}}$, stellar mass $M_{*}$, stellar radius $R_{*}$, and the impact parameter, the ratio of the tidal radius $r_{\mathrm{t}} \equiv R_{\mathrm{S}}\left(M_{\mathrm{h}} / M_{*}\right)^{1 / 3}$ to the distance of the closest approach $r_{\mathrm{p}}$, as $\beta=r_{t} / r_{p}$ (Rees 1988).

When the debris from a disrupted star falls back to the SMBH, it first forms an elliptical accretion disk from which matter circularizes via a combination of mechanisms (Ramirez-Ruiz \& Rosswog 2009; Hayasaki et al. 2013; Dai et al. 2013; Guillochon et al. 2014). The temporal evolution of the fallback rate $\dot{M}$ is thought to be characterized by a range of power-law values $\sim t^{-n}$, where $n$ depends on the star's structure and the impact parameter $\beta$ (Lodato et al. 2009; Guillochon \& Ramirez-Ruiz 2013). The conversion of the fallback into light is not expected to

\footnotetext{
$\overline{16}$ http://cosmo.nyu.edu/afterglowlibrary/boxfit2011.html
}

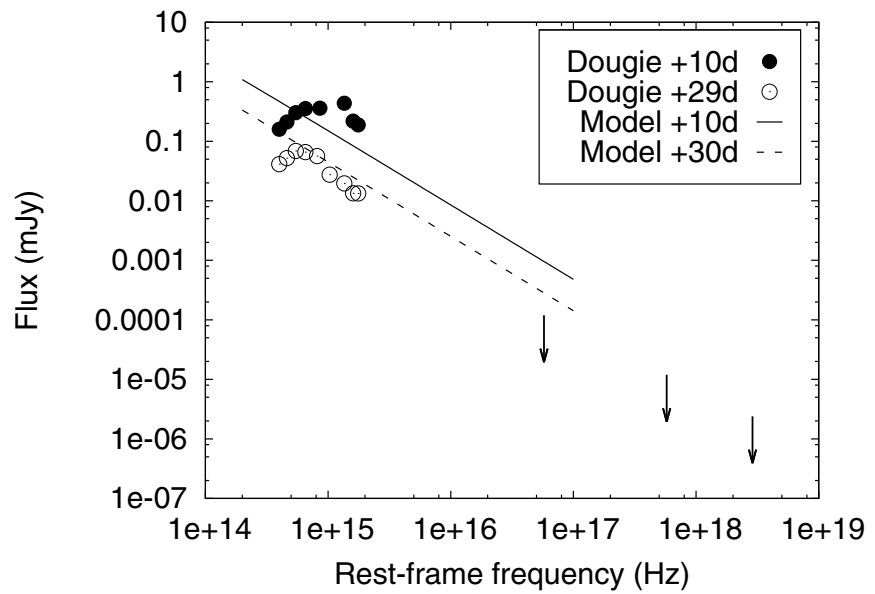

Figure 11. Predicted synchrotron spectra of the $\theta_{\mathrm{obs}}=1.6 \theta_{\mathrm{j}}$ orphan afterglow model in Figure 10 compared with the observed optical-UV SEDs (filled symbols). The X-ray upper limits calculated from the integrated Swift/XRT observations are indicated by the downward arrows.

directly follow $\dot{M}$, especially for individual bands (Strubbe \& Quataert 2009; Lodato \& Rossi 2011). As gas accretes onto the black hole, radiation pressure may expel some fraction of the mass if the accretion rate exceeds the Eddington limit of the black hole, $L_{\mathrm{Edd}}=4 G M_{\mathrm{h}} m_{\mathrm{p}} c / \sigma_{\mathrm{T}}$. When this condition is satisfied, a wind may be produced that carries a significant amount of kinetic and thermal energy (Strubbe \& Quataert 2009; Lodato \& Rossi 2011). For cases in which the accretion rate remains sub-Eddington, the disk component likely becomes the dominant source of radiation, peaking in the far UV to soft X-ray bands; however, previous TDE candidates have shown evidence of significant reprocessing of the emergent light into longer wavelengths with an SED characterized by a single blackbody, rather than the sum of blackbodies expected for an accretion disk (Gezari et al. 2012; Guillochon et al. 2014).

Even in the observed bands, which do not include the peak in the SED at early times (see Figure 4), the peak luminosity of Dougie, $L_{\text {peak }} \gtrsim 5 \times 10^{44} \mathrm{erg} \mathrm{s}^{-1}$, is larger than the Eddington limit of a $10^{7} M_{\odot}$ black hole. Additionally, Dougie's time of peak is significantly shorter than the time of peak predicted from $\dot{M}$ alone, which, for a $10^{6} M_{\odot}$ black hole, is on the order of a month for main-sequence stars (Guillochon \& Ramirez-Ruiz 2013). Both of these aspects suggest that if Dougie is indeed a tidal disruption event, its observational appearance near peak must be dominated by a wind component whose functional form may not directly reflect $\dot{M}$.

To model Dougie, we modified the TDEFit code initially described in Guillochon et al. (2014) to include a wind component that can carry a significant fraction of the accretion energy. The appearance of a TDE when its accretion rate exceeds Eddington has been explored by a number of authors (Strubbe \& Quataert 2009; Lodato \& Rossi 2011; Coughlin \& Begelman 2014). For super-Eddington accretion rates, these models presume a fraction of the incoming mass is ejected in the form of a wind, which moves out at some velocity that is comparable to the orbital velocity at the tidal disruption radius. If the majority of the energy carried by the wind is internal, the radiative output can be significantly less than the energy input, as much of the energy will be expended as work as the wind expands (Strubbe \& Quataert 2009). If the excess energy is instead mostly carried kinetically, but then dissipates near the photosphere, the radiative output can be comparable to the energy input. 


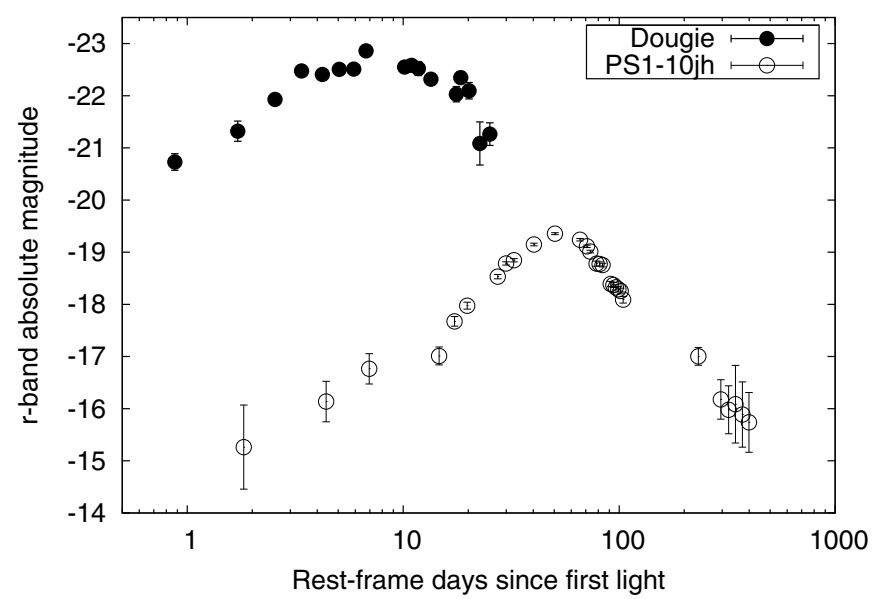

Figure 12. $r$-band absolute light curves of Dougie (filled circles) and PS1-10jh (open circles), another TDE-candidate (Gezari et al. 2012). For the latter, the redshift of $z=0.1696$ was applied to correct for time dilation and distance.

While these works provide descriptions of the relevant wind physics in the decline phase, they do not provide formalisms that are general enough to model Dougie over its full evolution: rise, peak, and decline. Additionally, each model has a particular prediction for the power-law relationship between $M$ and the photosphere's properties. As an example, Dougie shows a clear decrease in temperature as a function of time after peak (Figure 4), whereas Strubbe \& Quataert (2009) and Lodato \& Rossi (2011) predict a temperature increase until the event drops below the Eddington limit. Coughlin \& Begelman (2014), by contrast, do predict a slight temperature decrease after peak.

Figure 12 shows the comparison of the $r$-band LC of Dougie with that of another TDE-candidate, PS1-10jh (Gezari et al. 2012). It is seen that the two events had markedly different LCs: Dougie showed a much faster and more energetic outburst than PS1-10jh. Modeling of PS1-10jh has shown that a standard thin disk alone is not capable of fitting the event's LC (Guillochon et al. 2014), but that a large fraction of the disk's light needs to be intercepted by a reprocessing region to adequately match observations. We propose that the reprocessing region intercepts a fraction, $f_{\text {rep }}$, of the disk radiation. The reprocessing region is likely to be hydrostatic when the accretion rate is sub-Eddington, but to become dynamic and unbound when the Eddington limit is exceeded. The subsequent dynamic expansion then releases energy that originates from a combination of the radiation from the disk and radiation from the expanding reprocessing region,

$$
L=\left(1-f_{\text {rep }}\right) L_{\text {disk }}+L_{\text {rep }}
$$

We assume $L_{\text {disk }}$ is capped at the Eddington luminosity, and $L_{\text {rep }}$ is equal to the fraction of reprocessed disk light plus a fraction $f_{\text {out }}$ of the Eddington excess,

$$
L_{\text {rep }}=f_{\text {rep }} L_{\text {disk }}+\eta f_{\text {out }}\left(\dot{M}-\dot{M}_{\text {Edd }}\right) c^{2},
$$

where we have presumed that the maximum amount of energy released in the form of an outflow is given by the energy release at the innermost stable circular orbit (ISCO), $\eta c^{2}$, where the black hole efficiency $\eta$ depends solely on the black hole's spin parameter $a_{\text {spin. }}$. As in Guillochon et al. (2014), we do not presume an a priori time dependence of the photosphere on $M$, its optical depth $\tau$, its size $R_{\mathrm{ph}}$, or its temperature $T_{\mathrm{ph}}$, but rather leave these as free parameters. Because this model

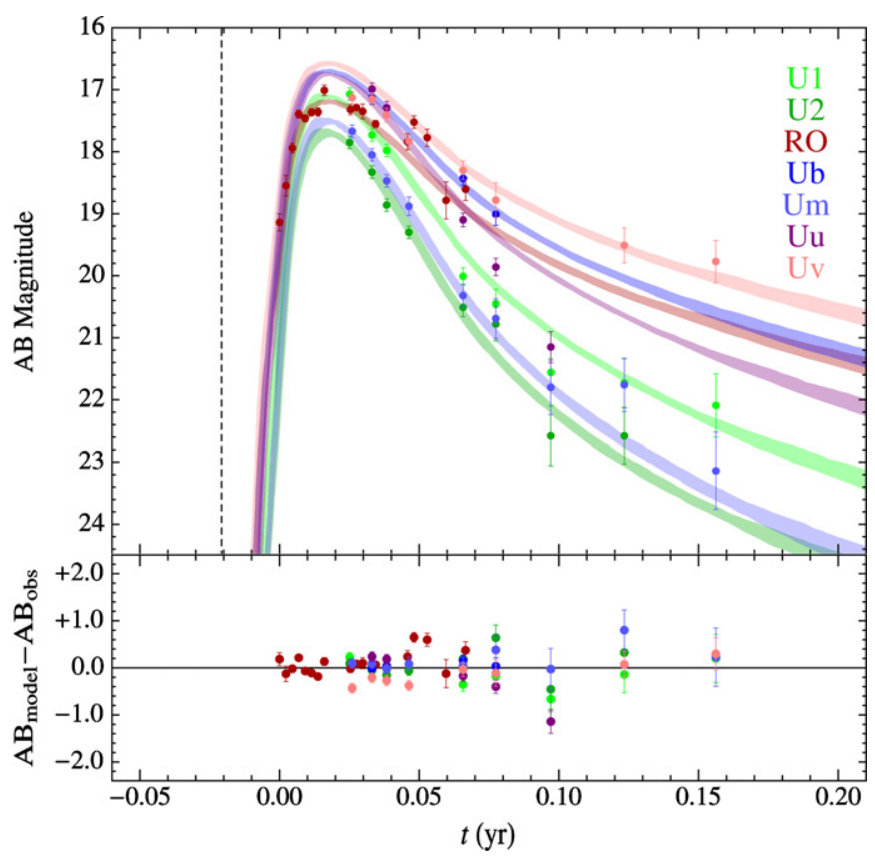

Figure 13. Results of maximum-likelihood analysis performed by TDEFit. The figure shows the Swift and ROTSE-IIIb photometry as compared to the $1 \sigma$ scatter of models with the highest likelihood. The top panel shows AB magnitudes of the data (dots) and models (shaded regions), while the bottom panel shows the difference between the data and the best-fit model.

does not presume a particular power-law relationship between $\dot{M}$ and the reprocessing region's properties, the model space includes the specific power-law index proposed in Strubbe \& Quataert (2009) and Lodato \& Rossi (2011), which would be favored by the Markov Chain Monte Carlo optimization if they are able to reproduce Dougie's observed evolution. One simplification made here is that we presume the power-law relationship between $\dot{M}, R_{\mathrm{ph}}$, and $T_{\mathrm{ph}}$ is constant throughout the event, regardless of whether the event is above or below the Eddington limit, whereas Strubbe \& Quataert (2009) and Lodato \& Rossi (2011) advocated a transition at the Eddington limit. We find that such a transition is not necessary to produce satisfactory fits (Figure 13), but relaxing this assumption may improve fit quality.

Beside the parameters described above $\left(M_{h}, M_{*}, \beta, a_{\mathrm{spin}}, R_{\mathrm{ph}}\right.$, $\tau$, and $f_{\text {out }}$ ) the model also includes the following additional parameters (see Guillochon et al. 2014 for more complete description): the power-law index $l$ in the relationship $R_{\mathrm{ph}} \propto$ $\dot{M}^{l}$; the disk inclination angle $\phi(\phi=0$ indicating face-on); the disk viscosity parameter $\mathcal{V}$; the polytropic index $\gamma$ of the disrupted star (either $5 / 3$ or $4 / 3$ ); the hydrogen column density $N_{H}$ within the host galaxy; and the reddening-law parameter $R_{V}$. We assumed that the time lag between the disruption and the first detection is $t_{\text {off }}$ (in days), and we added $\sigma_{v}$ variance (in magnitudes) to the model LCs.

\subsubsection{Properties of Highest-likelihood TDE Models}

In Table 4, we show the median values resulting from our maximum-likelihood analysis. As expected, the short peak timescale and high luminosity of Dougie favors a low-mass black hole, although there is very large scatter in the favored black hole mass $\left(\log _{10} M_{\mathrm{h}}=5.29_{-0.45}^{+0.91}\right)$ so that the black hole mass might be as large as $\approx 10^{6} M_{\odot}$. If we assume that the host galaxy's light is dominated by a bulge component, the black hole mass it would possess based on the Häring \& Rix (2004) relation 
Table 4

Parameters of Highest Likelihood TDE Models

\begin{tabular}{lcccc}
\hline \hline Parameter $^{\mathrm{a}}$ & Units & Prior & Allowed Range & Value $^{\mathrm{b}}$ \\
\hline$t_{\text {off }}$ & days & Flat & $-10^{3} \leqslant x \leqslant 10^{3}$ & $7.6_{-1.0}^{+1.3}$ \\
$\log _{10} M_{*}$ & $M_{\odot}$ & Kroupa & $-3 \leqslant x \leqslant 2$ & $-0.098_{-0.484}^{+0.449}$ \\
$\gamma$ & $\ldots$ & Flat & $4 / 3$ or $5 / 3$ & $5 / 3(99.7 \%)$ \\
$\log _{10} M_{\mathrm{h}}$ & $M_{\odot}$ & Flat & $4 \leqslant x \leqslant 8.6$ & $5.29_{-0.45}^{+0.91}$ \\
$\beta$ & $\ldots$ & $\beta^{-2}$ & $0.5 \leqslant x \leqslant 4$ & $0.97_{-0.15}^{+0.19}$ \\
$a_{\text {spin }}$ & $\ldots$ & Flat & $0 \leqslant x \leqslant 0.998$ & $0.64_{-0.45}^{+0.29}$ \\
$\log _{10} \mathcal{V}$ & $\ldots$ & Flat & $-4 \leqslant x \leqslant 0$ & $-0.19_{-0.19}^{+0.13}$ \\
$\phi$ & radians & Flat & $0 \leqslant x \leqslant \pi / 2$ & $0.51_{-0.36}^{+0.35}$ \\
$\log _{10} \tau$ & $\ldots$ & Flat & $-6 \leqslant x \leqslant 6$ & $3.1_{-2.4}^{+2.1}$ \\
$l$ & $\ldots$ & Flat & $0 \leqslant x \leqslant 4$ & $0.16_{-0.06}^{+0.06}$ \\
$\log _{10} R_{\text {ph } 0}$ & $\ldots$ & Flat & $-4 \leqslant x \leqslant 4$ & $0.98_{-0.26}^{+0.11}$ \\
$\log _{10} f_{\text {out }}$ & $\ldots$ & Flat & $-4 \leqslant x \leqslant 0$ & $-0.16_{-0.29}^{+0.12}$ \\
$R_{\mathrm{V}}$ & $\ldots$ & Flat & $2 \leqslant x \leqslant 10$ & $6.1_{-2.7}^{+2.7}$ \\
$\log _{10} N_{\mathrm{H}}$ & $\mathrm{cm}$ & Flat & $17 \leqslant x \leqslant 23$ & $19_{-1.3}^{+1.4}$ \\
$\sigma_{\mathrm{V}}$ & $\ldots$ & Flat & $0 \leqslant x \leqslant 1$ & $0.24_{-0.04}^{+0.04}$ \\
\hline
\end{tabular}

Notes.

a See Guillochon et al. (2014) for more detailed description.

${ }^{b}$ Median value, with ranges corresponding to $1 \sigma$ spread from median.

would be $\approx 10^{7} M_{\odot}$; however, as the galaxy is Sb-type, its bulge fraction may be as low as $20 \%$ (Binney \& Merrifield 1998), suggesting a central black hole mass of a few $10^{6} M_{\odot}$. This value is within the scatter of the black hole mass distribution found here. Another possibility is that the tidal disruption occurred about a secondary, smaller black hole in the process of merging with galaxy's primary black hole, which would also explain the TDE's slight offset from the host's center.

Lower-mass $\left(10^{5}-10^{6} M_{\odot}\right)$ black holes, even off-center ones, although rare, are not unprecedented. A good example is in NGC 3341, where recent merging resulted in two off-center nuclear sources (at $\sim 5$ and $\sim 8 \mathrm{kpc}$ from the center of the massive disk galaxy), one of them being a Seyfert-2 nucleus (Barth et al. 2008). Another lower-mass accreting $\mathrm{BH}\left(\sim 10^{6} M_{\odot}\right)$ was recently discovered in the central region of the dwarf starburst galaxy Henize 2-10 (Reines \& Deller 2012). The more recent detection of a radio outburst from the ultra-luminous X-ray source HLX-1 in ESO 243-49 may be due to a jet ejection from an intermediate-mass $\left(10^{3}-10^{4} M_{\odot}\right) \mathrm{BH}$ (Webb et al. 2014). As illustrated by these examples, the off-center position $(\sim 4 \mathrm{kpc})$ of Dougie, as well as the BH mass derived in the TDE-model, are not unrealistic, and may be consistent with the TDE-hypothesis.

A number of differences in Dougie's highest-likelihood parameters are apparent when comparing to fits of PS1-10jh. The best-fitting photosphere power-law index we find is small, $l=0.16$. This parameter is related to the temperature power-law index by the expression $2 l+4 m=1$ (Guillochon et al. 2014). This implies that $T_{\mathrm{ph}} \propto \dot{M}^{0.17}$, close to the expectation of $\dot{M}^{1 / 4}$ for a blackbody the luminosity of which is proportional to $\dot{M}$ with a fixed $R_{\text {ph. }}$. Figure 14 shows that the photosphere grows to a few $10^{15} \mathrm{~cm}$ (top panel), at velocity comparable to the escape velocity at $2 r_{\mathrm{p}}$ (middle panel), the terminal velocity for a wind launched from that location (Strubbe \& Quataert 2009). The photosphere grows beyond the distance to which both the bound debris and unbound tidal tail have traveled since the time of disruption, suggesting that it must be continually accelerated; however, as the area of the photosphere increases more slowly
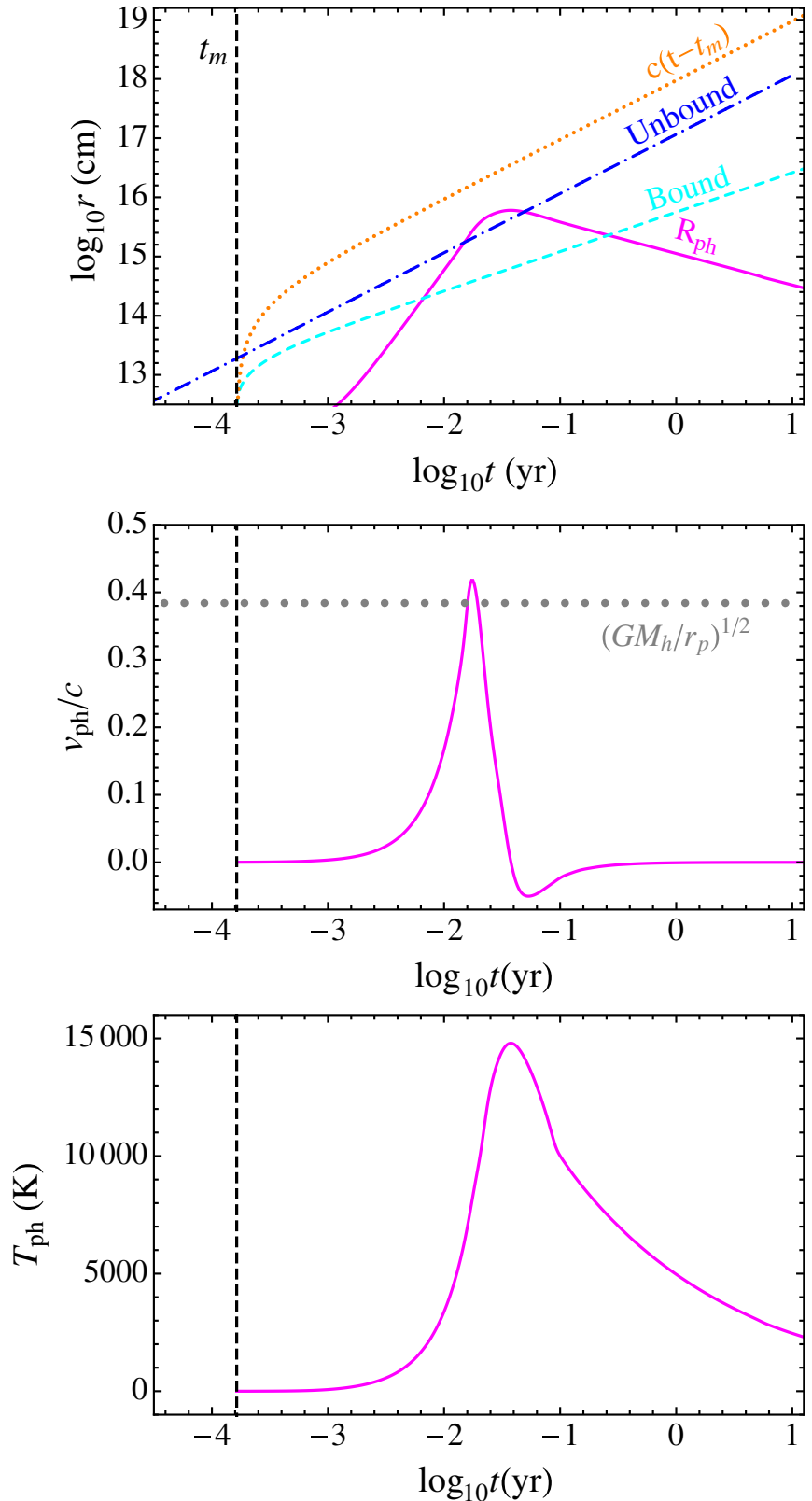

Figure 14. Evolution of various quantities as functions of time for the TDE scenario for the highest-likelihood match to Dougie, where the vertical dashed times indicate the time of return of the most bound material $t_{\mathrm{m}}$. The top panel shows distance of various features as a function of time: the bound debris (cyan dashed), the unbound tidal tail (blue dot-dashed), the light travel distance since the time of disruption (orange dotted), and the location of the wind/ reprocessing photosphere (magenta solid). The middle panel shows the velocity of the photosphere $v_{\mathrm{ph}}$, where the gray dotted line shows the escape velocity at twice the periapse distance. The bottom panel shows the temperature of the photosphere $T_{\mathrm{ph}}$.

than $\dot{M}, T_{\text {ph }}$ also rapidly increases near peak and then cools off slowly at later times (Figure 14, bottom panel). For PS1-10jh, it was found that the reprocessing region's temperature was inversely related to $\dot{M}$ (Guillochon et al. 2014).

The models also favor nearly $100 \%$ conversion of both the kinetic energy from the wind and radiative energy from the disk into energy radiated by the reprocessing photosphere (i.e., $f_{\text {out }} \sim 1$ and $\tau \rightarrow \infty$ ), suggesting that the wind component is completely dominant. This conclusion is bolstered by the strong upper limits in the X-rays from Swift (see Section 2.2) that suggest $<1 \%$ of the radiative output emerges with energies 


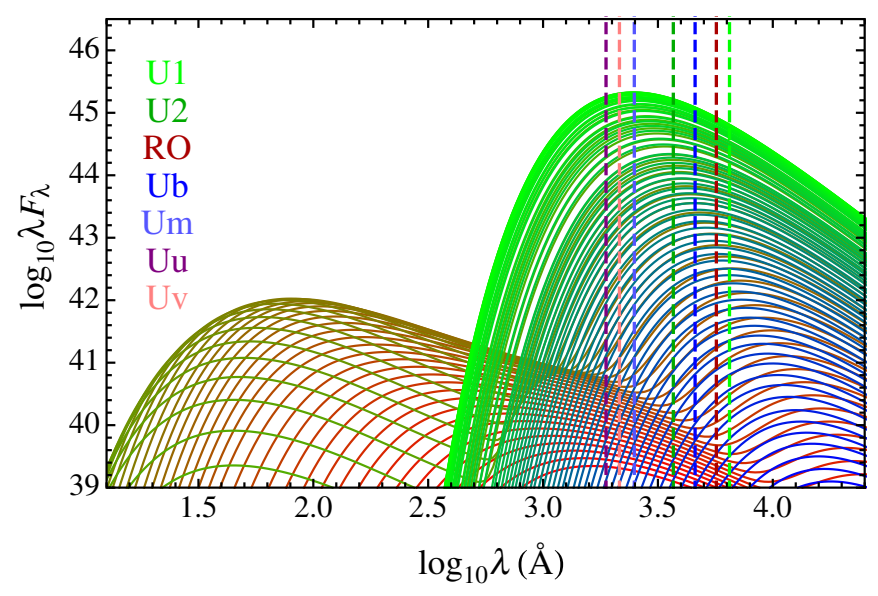

Figure 15. Spectral energy distributions (SEDs) as a function of time for the highest-likelihood fit to Dougie. Red curves correspond to early times, green curves correspond to the flare at peak luminosity, and blue curves correspond to late times. The vertical dashed lines show the centroid of each filter used to observe Dougie, color-coded to match the filter designations in the top left.

above $200 \mathrm{eV}$. Our highest likelihood models suppress the total $\mathrm{X}$-ray output to $<10^{42} \mathrm{erg} \mathrm{s}^{-1}$ (Figure 15). By contrast, the reprocessing zone in PS1-10jh was found to only intercept $\sim$ one-third of the disk's radiative output. To see if such a high conversion factor was necessary, we performed a test TDEFit run in which $f_{\text {out }}$ was fixed to 0.1 , which yielded a poor fit and tended to even lower black hole masses $\left(M_{\mathrm{h}}<10^{5}\right)$. The low Xray flux also suggests that if a jet were produced (e.g., De Colle et al. 2012b), it was at the very least not pointed toward Earth, and the high conversion ratio into the reprocessing zone may be the result of the jet being intercepted by a thick, super-Eddington accretion flow (Tchekhovskoy et al. 2014).

Aside from these differences, the favored stellar mass $\left(M_{*}=\right.$ $\left.0.8 M_{\odot}\right)$, impact parameter $(\beta=0.97$, indicating a full disruption for the favored $\gamma)$, black hole spin $(a=0.64)$, and viscous parameter $(\mathcal{V}=0.65)$ are all typical values expected for a main-sequence disruption, suggesting that this event would be representative of disruptions about lower-mass black holes if it is in fact a tidal disruption.

\section{SUMMARY}

As a summary, we draw the following conclusions.

1. We discovered an OT (nicknamed Dougie) that was most likely of extragalactic origin. It appeared on top of a faint galaxy having $z=0.191$ redshift corresponding to $D \approx 900 \mathrm{Mpc}$ distance. Our follow-up observations in the optical and UV bands revealed that the LC of the transient showed a quick rise (with rise time of $\sim 10$ days) followed by a subsequent decline resulting in $\mathrm{a} \approx 1$ month long observability with our resources. The observed LC suggested a peak luminosity of $\approx 5 \times 10^{44} \mathrm{erg} \mathrm{s}^{-1}$, which is similar to those of the most luminous SLSNe. Simultaneous $\mathrm{X}$-ray observations with Swift resulted in no detected X-ray emission on the co-added XRT frames spanning the whole duration of the follow-up observations.

2. Our spectroscopic follow-up observations showed that the spectra of the transient were unusual: between 4000 and $9000 \AA$ it did not show any spectral feature that could be attributed to the transient, during the whole observed interval. The weak narrow features in the late-time spectra were identified as due to the host galaxy. At early times the spectra consisted of a hot, blue continuum $(T \approx 16,000 \mathrm{~K})$, which later cooled down, but could not be described as a single blackbody from the UV to the red. The softening of the spectra suggested a cooling, expanding, SN-like ejecta, but the lack of spectral features during the entire observed time domain argued against the $\mathrm{SN}$-hypothesis.

3. The proposed host galaxy, SDSS J120847.77+4320.1, is a faint Sb-type galaxy, without any previously detected $\mathrm{UV} / \mathrm{X}$-ray emission, and without any sign of ongoing star formation. The transient appeared $\approx 1.3$ arcsec off-center, corresponding $\approx 3.9 \mathrm{kpc}$ linear distance from the photocenter of the host $(2 \sigma)$.

4. Despite the similar peak luminosity, it is unlikely that the transient was a SLSN. Traditional SN models based on radioactive decay are ruled out because of the order-ofmagnitude difference between the required amount of ${ }^{56} \mathrm{Ni}$ mass $\left(\approx 15 M_{\odot}\right)$ and ejecta mass $\left(\approx 1 M_{\odot}\right.$, from LC rise time). The magnetar-powered and the CSM-interactionpowered SLSN models can be tweaked to produce a similar LC, but the difference between the usual spectra of these kind of transients and that of Dougie does not support the SLSN hypothesis. Also, the lack of ongoing star formation within the host galaxy is not typical for galaxies producing $\mathrm{H}$-poor SLSNe, as those SLSNe tend to appear in metalpoor hosts showing intense star formation.

5. Merging neutron stars that can produce fast, luminous transients, where the remnant collapse is halted when a rapidly rotating, hypermassive magnetar is produced, usually result in LCs that evolve much faster than Dougie. Their predicted rise times, $t_{\text {rise }} \sim 1-2$ days (Metzger $\&$ Piro 2014), are in contrast to the observed $\sim 10$ days rise time of Dougie.

6. Based on the similarity of the hot featureless spectra to the early spectra of GRB-SNe, afterglow models by van Eerten et al. (2012) were fit to the LC and SEDs of Dougie. Both the shape and the peak of the LC could be explained by a jetinduced afterglow having parameters more or less similar to those derived by Cenko et al. (2013) for PTF11-agg. All such models, however, fail to reproduce the observed SED evolution.

7. The tidal disruption scenario was explored by fitting the event to an amended version of the model presented in Guillochon et al. (2014). The TDE model yielded a good fit to the photometric and spectral evolution of the flare, with the highest-likelihood models suggesting a disruption of a solar-mass star by a black hole. The BH mass turned out to be on the low end of predictions for the associated host galaxy. Due to the slight offset of the flare from the host's center, this may be attributable to a recent merger of a lower-mass galaxy with the host. The TDE model also finds that the flare must have been very super-Eddington at peak with a near full conversion of energy released at the ISCO into energy radiated at its photosphere. As no X-rays were detected from the flare, there is no direct evidence for the existence of a jet, suggesting that the jet was "smothered" by the super-Eddington accretion flow, which is consistent with the near-full conversion of accretion energy into radiative output. The other parameters of the disruption yielded by our analysis are as expected of a typical disruption. We thus conclude that Dougie could represent a canonical TDE about lower-mass central black holes. 
We thank the anonymous referee for the thorough report, which was helpful while revising the first version of this paper. This work has been supported by NSF grant AST 11-09881 (UT, PI Wheeler), Hungarian OTKA grant NN-107637 (Szeged, PI Vinko), NSF grant AST-0847563 (UCSC, PI Ramirez-Ruiz), and the David and Lucile Packard Foundation (ERR). G.H.M. is supported by NSF grant AST 09-07903 (CfA, PI Kirshner). J.G. is supported by Einstein grant PF3-140108. E.C. was supported by the University of Texas at Austin Graduate Scholl Power's Fellowship, and is currently supported by the Enrico Fermi Institute via the Enrico Fermi Postdoctoral Fellowship. ROTSE-III has been supported by NASA grant NNX-08AV63G, NSF Grant PhY-0801007, the Australian Research Council, the University of New South Wales, the University of Texas, and the University of Michigan. The Marcario Low Resolution Spectrograph is named for Mike Marcario of the High Lonesome Optics, who fabricated several optics for the instrument but died before its completion. The LRS is a joint project of the Hobby-Eberly Telescope partnership and the Instituto de Astronomía de la Universidad Nacional Autónoma de México. We acknowledge the HET Resident Astronomer Team (M. Shetrone, S. Odewahn, J. Caldwell, S. Rostopchin) for their work and support during the spectroscopic follow-up observations. The NASA ADS and NED services were extensively used during the entire project, and the availability of these services are also gratefully acknowledged.

\section{REFERENCES}

Arcavi, I., Gal-Yam, A., \& Sullivan, M. 2014, ApJ, 793, 38

Barnes, J., \& Kasen, D. 2013, ApJ, 775, 18

Barth, A. J., Bentz, M. C., Greene, J. E., \& Ho, L. C. 2008, ApJL, 683, L119

Benetti, S., Nicholl, M., Cappellaro, E., et al. 2014, MNRAS, 441, 289

Binney, J., \& Merrifield, M. 1998, Galactic Astronomy (Princeton, NJ: Princeton Univ. Press)

Cenko, S. B., Kulkarni, S. R., Horesh, A., et al. 2013, ApJ, 769, 130

Chatzopoulos, E., Wheeler, J. C., Vinko, J., et al. 2011, ApJ, 729, 143

Chen, T.-W., Smartt, S. J., Jerkstrand, A., et al. 2014, arXiv:1409.7728

Chornock, R., Berger, E., Gezari, S., et al. 2014, ApJ, 780, 44

Coughlin, E. R., \& Begelman, M. C. 2014, ApJ, 781, 82

Dai, L., Escala, A., \& Coppi, P. 2013, ApJL, 775, L9

De Colle, F., Granot, J., López-Cámara, D., \& Ramirez-Ruiz, E. 2012a, ApJ, 746,122

De Colle, F., Guillochon, J., Naiman, J., \& Ramirez-Ruiz, E. 2012b, ApJ, 760,103

De Colle, F., Ramirez-Ruiz, E., Granot, J., \& Lopez-Camara, D. 2012c, ApJ, 751,57

Gal-Yam, A. 2012, Sci, 337, 927

Gal-Yam, A., Mazzali, P., Ofek, E. O., et al. 2009, Natur, 462, 624

Gezari, S., Chornock, R., Rest, A., et al. 2012, Natur, 485, 217
Gezari, S., Halpern, J. P., Grupe, D., et al. 2009, ApJ, 690, 1313

Granot, J., Panaitescu, A., Kumar, P., \& Woosley, S. E. 2002, ApJL, 570, L61

Guillochon, J., Manukian, H., \& Ramirez-Ruiz, E. 2014, ApJ, 783, 23

Guillochon, J., \& Ramirez-Ruiz, E. 2013, ApJ, 767, 25

Häring, N., \& Rix, H. 2004, ApJL, 604, L89

Hayasaki, K., Stone, N., \& Loeb, A. 2013, MNRAS, 434, 909

Hill, G. J., Nicklas, H. E., MacQueen, P. J., et al. 1998, Proc. SPIE, 3355, 375

Holoien, T. W.-S., Prieto, J. L., Bersier, D., et al. 2014, MNRAS, 445, 3263

Kalberla, P. M. W., Burton, W. B., Hartmann, D., et al. 2005, A\&A, 440, 775

Kasen, D., \& Bildsten, L. 2010, ApJ, 717, 245

Kinney, A. L., Calzetti, D., Bohlin, R. C., et al. 1996, ApJ, 467, 38

Lee, W. H., \& Ramirez-Ruiz, E. 2007, NJPh, 9, 17

Leloudas, G., Schulze, S., Kruehler, T., et al. 2014, arXiv:1409.8331

Lodato, G., King, A. R., \& Pringle, J. E. 2009, MNRAS, 392, 332

Lodato, G., \& Rossi, E. M. 2011, MNRAS, 410, 359

Lunnan, R., Chornock, R., Berger, E., et al. 2013, ApJ, 771, 97

Lunnan, R., Chornock, R., Berger, E., et al. 2014, ApJ, 787, 138

Metzger, B. D., Martínez-Pinedo, G., Darbha, S., et al. 2010, MNRAS, 406,2650

Metzger, B. D., \& Piro, A. L. 2014, MNRAS, 439, 3916

Miller, A. A., Chornock, R., Perley, D. A., et al. 2009, ApJ, 690, 1303

Neill, J. D., Sullivan, M., Gal-Yam, A., et al. 2011, ApJ, 727, 15

Nicholl, M., Smartt, S. J., Jerkstrand, A., et al. 2013, Natur, 502, 346

Ofek, E. O., Rabinak, I., Neill, J. D., et al. 2010, ApJ, 724, 1396

Oke, J. B., Cohen, J. G., Carr, M., et al. 1995, PASP, 107, 375

Oke, J. B., \& Gunn, J. E. 1982, PASP, 94, 586

Poole, T. S., Breeveld, A. A., Page, M. J., et al. 2008, MNRAS, 383, 627

Quimby, R. M. 2006, PhD thesis, The Univ. of Texas at Austin

Quimby, R. M., Aldering, G., Wheeler, J. C., et al. 2007, ApJL, 668, L99

Quimby, R. M., Kulkarni, S. R., Kasliwal, M. M., et al. 2011, Natur, 474, 487

Quimby, R. M., Yuan, F., Akerlof, C., \& Wheeler, J. C. 2013, MNRAS, 431, 912

Quimby, R. M., Yuan, F., Akerlof, C., Wheeler, J. C., \& Warren, M. S. 2012, AJ, 144,177

Ramirez-Ruiz, E., Granot, J., Kouveliotou, C., et al. 2005, ApJL, 625, L91

Ramirez-Ruiz, E., \& Rosswog, S. 2009, ApJL, 697, L77

Ramsey, L. W., Adams, M. T., Barnes, T. G., et al. 1998, Proc. SPIE, 3352, 34

Rees, M. 1988, Natur, 333, 523

Reines, A. E., \& Deller, A. T. 2012, ApJL, 750, L24

Roberts, L. F., Kasen, D., Lee, W. H., \& Ramirez-Ruiz, E. 2011, ApJL, 736, L21

Rosswog, S., Ramirez-Ruiz, E., \& Davies, M. B. 2003, MNRAS, 345, 1077

Sari, R., Piran, T., \& Narayan, R. 1998, ApJL, 497, L17

Schlegel, D. J., Finkbeiner, D. P., \& Davis, M. 1998, ApJ, 500, 525

Smith, N., Li, W., Foley, R. J., et al. 2007, ApJ, 666, 1116

Strubbe, L. E., \& Quataert, E. 2009, MNRAS, 400, 2070

Tchekhovskoy, A., Metzger, B., Giannios, D., et al. 2014, MNRAS, 437, 2744

van Eerten, H., van der Horst, A., \& MacFadyen, A. 2012, ApJ, 749, 44

van Eerten, H., Zhang, W., \& MacFadyen, A. 2010, ApJ, 722, 235

Webb, N. A., Godet, O., Wiersema, K., et al. 2014, ApJL, 780, L9

Woods, E., \& Loeb, A. 1999, ApJ, 523, 187

Woosley, S. E. 2010, ApJL, 719, L204

Wu, X.-F., Gao, H., Ding, X., et al. 2014, ApJL, 781, L10

Yu, Y.-W., Zhang, B., \& Gao, H. 2013, ApJL, 776, L40

Yuan, F. 2010, PhD thesis, Univ. of Michigan

Zhang, W., \& MacFadyen, A. 2009, ApJ, 698, 1261 